\title{
Myeloid mineralocorticoid receptor controls macrophage polarization and cardiovascular hypertrophy and remodeling in mice
}

\author{
Michael G. Usher, ${ }^{1}$ Sheng Zhong Duan, ${ }^{1,2,3}$ Christine Y. Ivaschenko, ${ }^{1}$ Ryan A. Frieler, ${ }^{4}$ \\ Stefan Berger, ${ }^{5}$ Günther Schütz, ${ }^{5}$ Carey N. Lumeng, ${ }^{1,6}$ and Richard M. Mortensen ${ }^{1,4,7}$
}

\begin{abstract}
1Department of Molecular and Integrative Physiology and 'Department of Internal Medicine, Nephrology Division, University of Michigan Medical School, Ann Arbor, Michigan, USA. ${ }^{3}$ Key Laboratory of Nutrition and Metabolism, Institute for Nutritional Sciences, Shanghai Institutes for Biological Sciences, Chinese Academy of Sciences, Shanghai, China. ${ }^{4}$ Department of Pharmacology, University of Michigan Medical School, Ann Arbor, Michigan, USA. ${ }^{5}$ German Cancer Research Center (DKFZ), Division Molecular Biology of the Cell I, Heidelberg, Germany.

${ }^{6}$ Department of Pediatrics and Communicable Diseases and 7Department of Internal Medicine,

Metabolism Endocrinology and Diabetes Division, University of Michigan Medical School, Ann Arbor, Michigan, USA.
\end{abstract}

\begin{abstract}
Inappropriate excess of the steroid hormone aldosterone, which is a mineralocorticoid receptor (MR) agonist, is associated with increased inflammation and risk of cardiovascular disease. MR antagonists are cardioprotective and antiinflammatory in vivo, and evidence suggests that they mediate these effects in part by aldosterone-independent mechanisms. Here we have shown that MR on myeloid cells is necessary for efficient classical macrophage activation by proinflammatory cytokines. Macrophages from mice lacking MR in myeloid cells (referred to herein as MyMRKO mice) exhibited a transcription profile of alternative activation. In vitro, MR deficiency synergized with inducers of alternatively activated macrophages (for example, IL-4 and agonists of PPAR $\gamma$ and the glucocorticoid receptor) to enhance alternative activation. In vivo, MR deficiency in macrophages mimicked the effects of MR antagonists and protected against cardiac hypertrophy, fibrosis, and vascular damage caused by L-NAME/Ang II. Increased blood pressure and heart rates and decreased circadian variation were observed during treatment of MyMRKO mice with L-NAME/Ang II. We conclude that myeloid MR is an important control point in macrophage polarization and that the function of MR on myeloid cells likely represents a conserved ancestral MR function that is integrated in a transcriptional network with PPAR $\gamma$ and glucocorticoid receptor. Furthermore, myeloid MR is critical for blood pressure control and for hypertrophic and fibrotic responses in the mouse heart and aorta.
\end{abstract}

\section{Introduction}

Mineralocorticoid receptor (MR) antagonists are important in the treatment of cardiovascular disease $(1,2)$ and have been shown to be beneficial in models of diabetic nephropathy (3), vascular fibrosis (4), atherosclerosis (5), and insulin resistance (6). Conversely, inappropriate excess of the MR agonist aldosterone is associated with increased cardiovascular disease and causes perivascular fibrosis and inflammation (7). Despite the close conceptual link between aldosterone action and the cardioprotective effects of MR antagonists, there remains significant evidence that $\mathrm{MR}$ antagonists have aldosteroneindependent mechanisms (8). In addition, eplerenone is effective in treating atherosclerotic mouse models that do not have elevations in aldosterone (5). The mechanisms of MR-mediated cardiovascular damage - in particular, the contributions of different cell types - are still poorly understood. We have investigated the contribution of myeloid cells to cardiovascular damage and hypertension and determined the role of MR in modulating macrophage phenotype.

Heterogeneity of macrophage polarization has recently been recognized as an important feature of diverse diseases including pulmonary fibrosis, tumor progression, angiogenesis, and obesity $(9,10)$. Macrophage responses fall within a spectrum of classically activated (referred to as M1 macrophages) and alternatively activated states

Authorship note: Michael G. Usher and Sheng Zhong Duan share first authorship. Conflict of interest: The authors have declared that no conflict of interest exists. Citation for this article: J Clin Invest. 2010;120(9):3350-3364. doi:10.1172/JCI41080.
$(\mathrm{AM} \phi)$, originally defined by the phenotypes induced by Th 1 and Th2 cytokines, respectively. Other stimuli, such as glucocorticoids through the glucocorticoid receptor (GR) or IL-10, produce alternatively activated macrophages with a mosaic of different and overlapping characteristics $(11,12)$. The regulation of AM $\phi$ and the importance of different subtypes in the development of inflammatory disease are poorly understood. Recent evidence has identified nuclear hormone receptors (PPPAR $\gamma$ and $-\delta$ ) that when activated (13-15) directly skew macrophages to an AM $\phi$ state, thereby improving metabolic function. GR activation also alters macrophage polarization, a phenotype that overlaps that of PPAR $\gamma$ activation but is not identical to it (16).

Activation of PPAR $\gamma$ by pioglitazone mimics the effects of MR antagonists in mitigating cardiac and vascular inflammation and fibrosis caused by mineralocorticoid excess and Ang II $(17,18)$. It was shown that the inhibition of Ang II-stimulated cardiac remodeling by pioglitazone required macrophage and not cardiac PPAR $\gamma(18)$. This suggested that macrophage activation and polarization are critical for cardiac function and inflammatory responses in Ang II-induced cardiac damage. Thus, we hypothesized that direct effects on inflammatory cells are an important mechanism of action of MR antagonists such as eplerenone and spironolactone and that macrophage MR directly opposes the action of PPAR $\gamma$ skewing the macrophage to classical activation. To test this hypothesis, we determined the direct effects of MR activation and inactivation on macrophage phenotype in vitro and in a mouse model of cardiovascular damage and remodeling. 

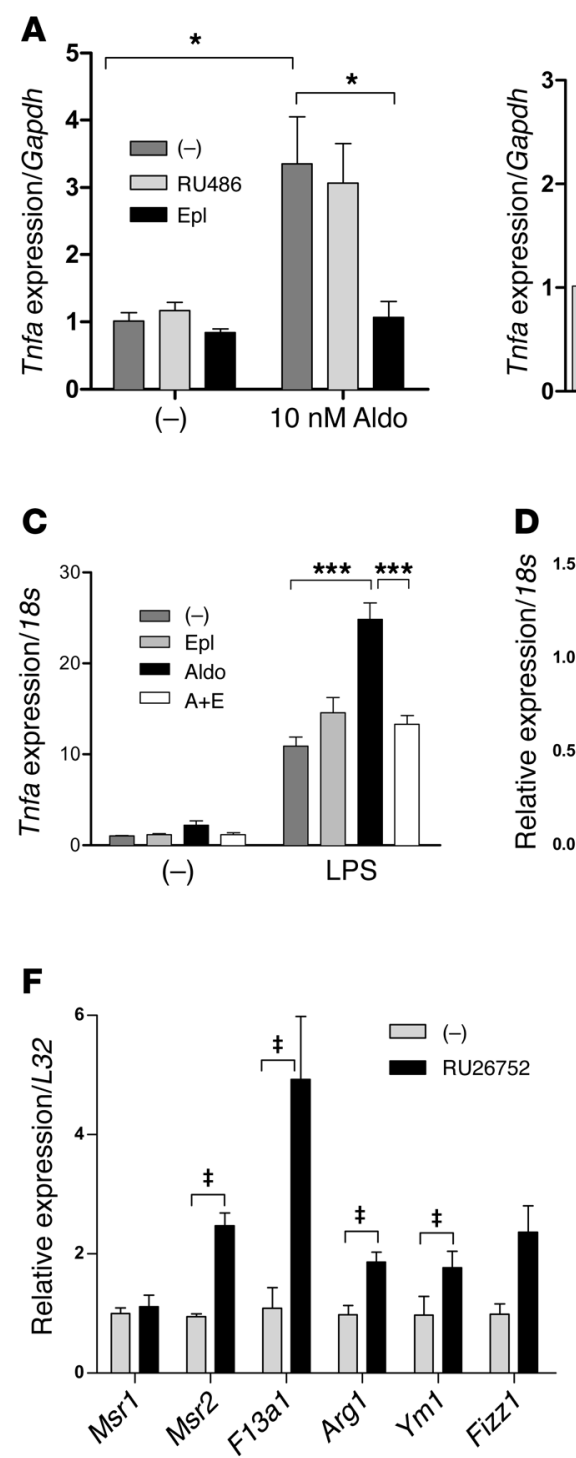
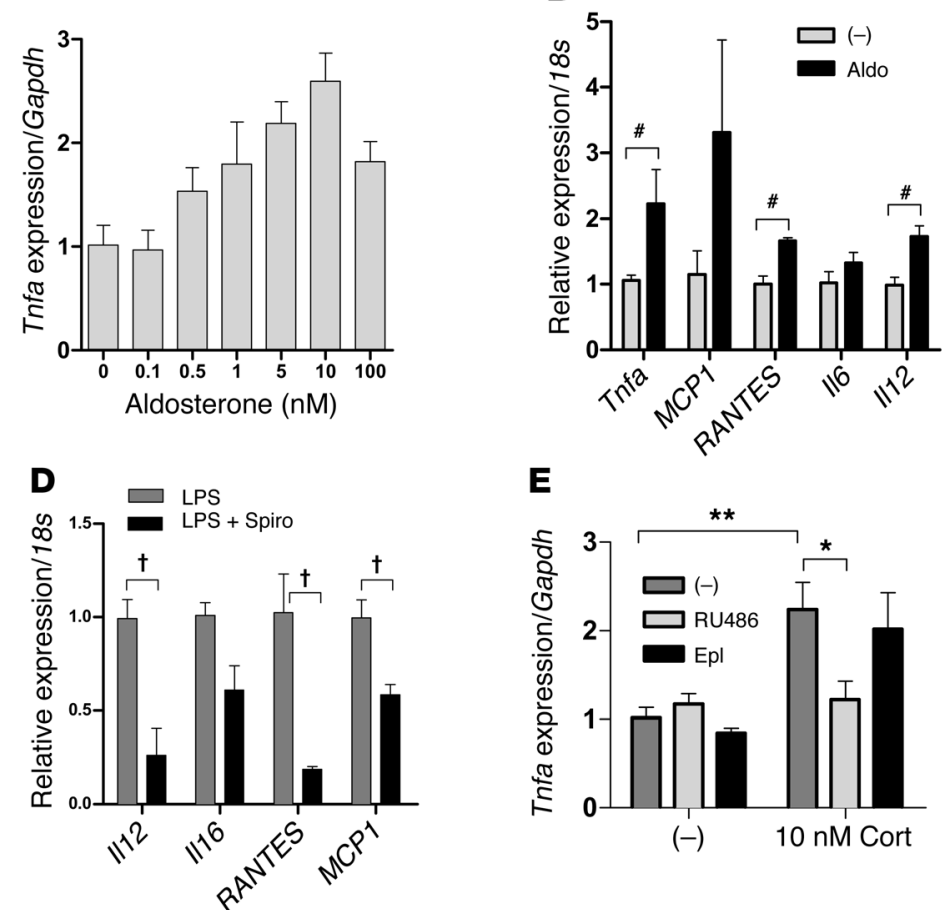
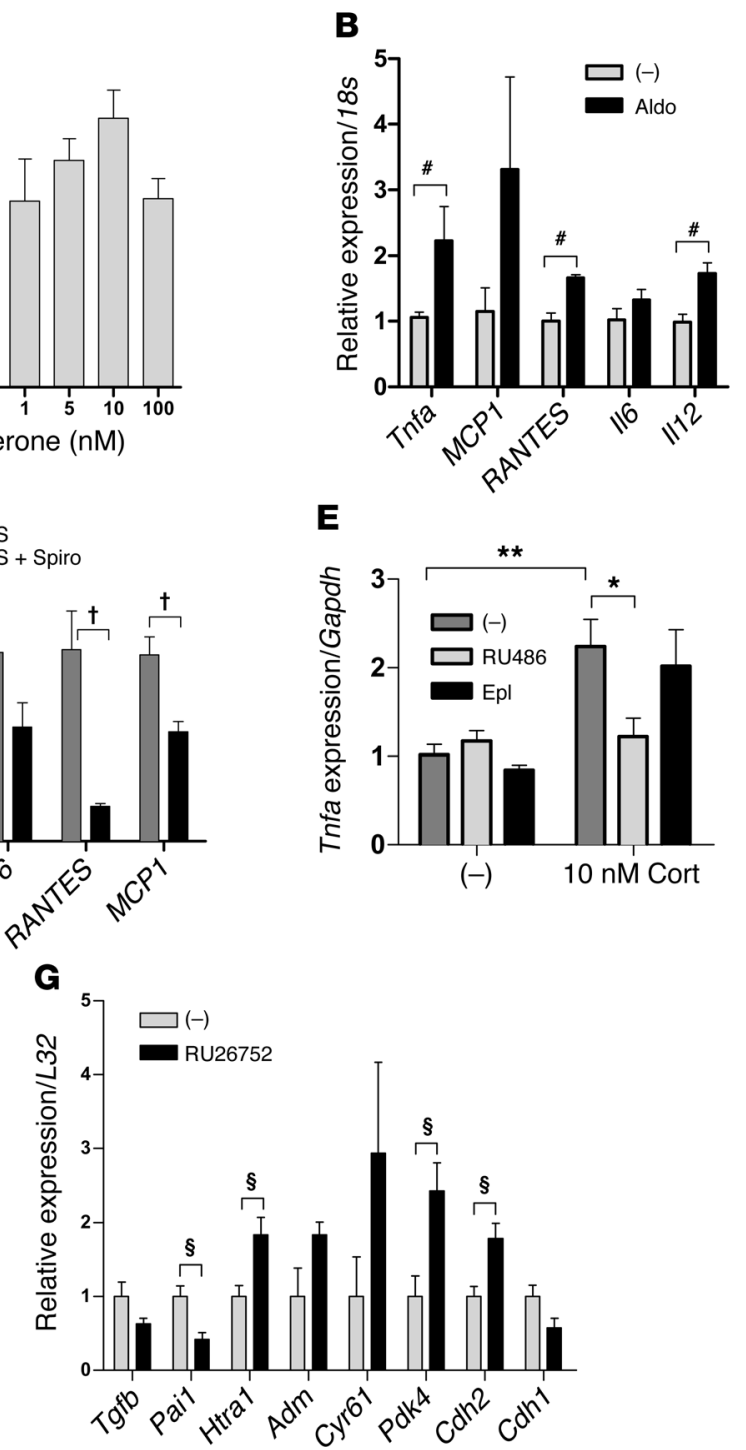

\section{Figure 1}

MR controls macrophage polarization. We studied the in vitro effects of MR agonists and antagonists in cultured PEMs. (A) Stimulation of TNF- $\alpha$ expression by aldosterone (Aldo; $10 \mathrm{nM}$ ) in steroid-depleted serum is abolished by MR but not GR antagonist. The dose response of Tnfa expression to different concentrations of aldosterone in steroid-depleted medium shows a high-affinity response consistent with MR. (B) Aldosterone (10 $\mathrm{nM}$ ) causes increases in M1 classically activated proinflammatory genes in steroid-depleted medium. (C) Aldosterone $(10 \mathrm{nM})$ increases the Tnfa response to LPS in steroid-depleted medium that is blocked by eplerenone (Epl; $5 \mu \mathrm{M})$. (D) In normal serum (10\% FBS), the MR antagonist spironolactone (Spiro; $5 \mu \mathrm{M}$ for 24 hours) decreases proinflammatory gene expression. (E) Corticosterone increases Tnfa expression in steroid-depleted medium that is blocked by the GR antagonist RU486 (5 $\mu \mathrm{M})$ but not eplerenone $(5 \mu \mathrm{M})$. (F) Twenty-four hours of $1 \mu \mathrm{M}$ RU26752, a specific MR antagonist, increases AM $\phi$ marker expression. (G) The MR antagonist RU26752 (1 $\mu \mathrm{M}$ ) increases some antifibrotic (Htra1, a TGF- $\beta$ inhibitor) and cardioprotective (e.g., Adm) genes and decreases some profibrotic genes (e.g., Pai1). ${ }^{\sharp} P<0.03,{ }^{\dagger} P<0.038, \ddagger P<0.034, \S P<0.025$, Benjamini-Hochberg correction; ${ }^{\star} P<0.05$, ${ }^{\star \star} P<0.01,{ }^{* \star *} P<0.001$ by 2 -way ANOVA with Bonferroni post-tests.

\section{Results}

MR controls macrophage polarization. To test the role of MR directly in macrophages, we investigated the effects MR agonists and antagonists on the in vitro phenotype in classical and alternative activation of the macrophages. Isolated peritoneal thioglycolateelicited macrophages (PEMs) cultured in charcoal/dextan-stripped media (C/D medium), to remove endogenous steroids, responded to aldosterone with an increase in the mRNA of a classical activation marker, Tnfa. This activation was inhibited by the MR antag- onist eplerenone but not the GR antagonist RU486 (Figure 1A). The dose response curve was also consistent with MR binding. Aldosterone induced other M1 markers as well (Figure 1B). LPS stimulation of M1 markers was similarly enhanced by aldosterone and blocked by MR antagonists (Figure 1C).

Most reports fail to show any expression of the glucocorticoidinactivating enzyme $11 \beta$ HSD2 (19), and we did not detect any $11 \beta H S D 2$ mRNA by PCR in PEMs (data not shown). Therefore, the ligand is often thought to be glucocorticoids, as in the hippocam- 
A

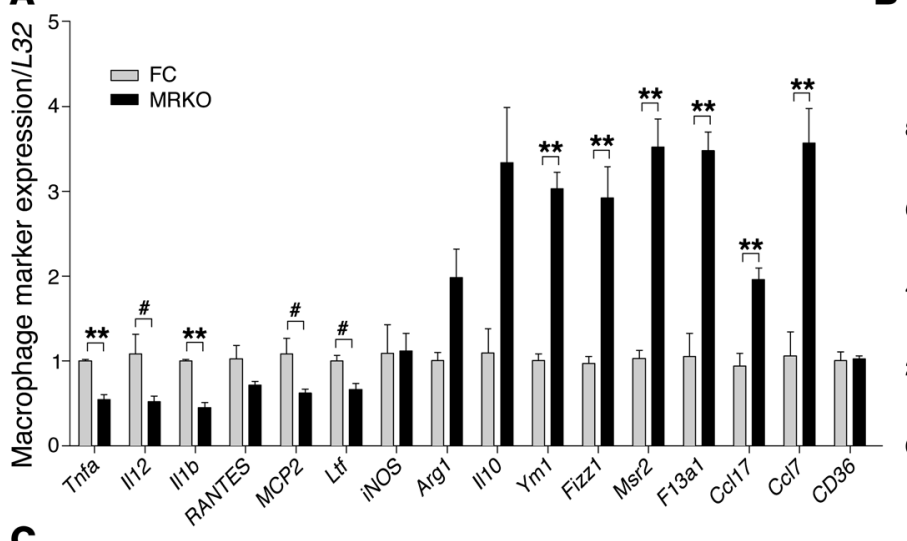

C
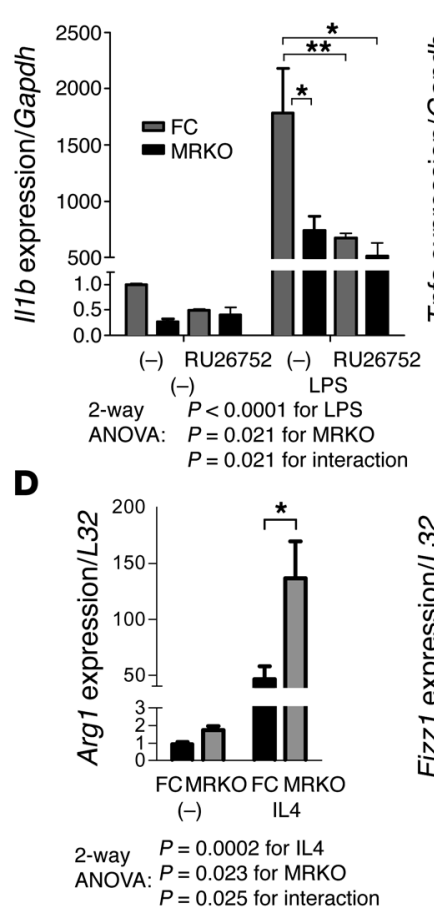
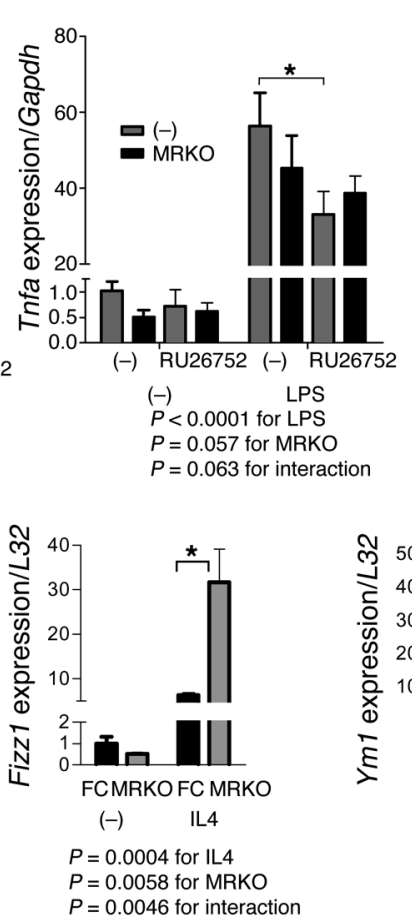
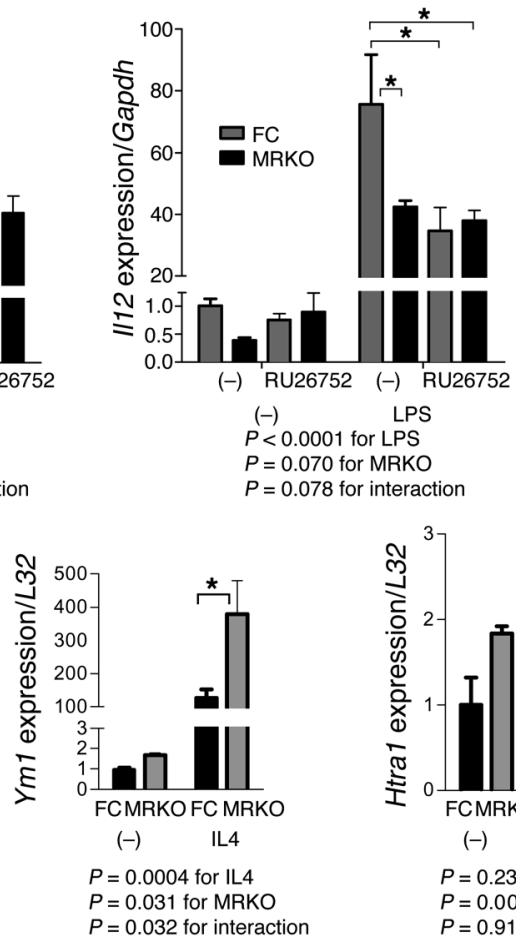

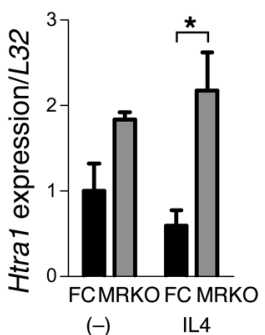

$P=0.23$ for interaction $P=0.0014$ for MRKO $P=0.91$ for IL4

Figure 2

MyMRKO causes alteration similar to that of MR antagonists in macrophages. PEMs were isolate from littermate FC or MyMRKO mice. (A) PEMs isolated from MyMRKO (MRKO) compared with FC mice show increased expression of AM $\phi$ markers and decreased M1 markers. (B) Differences in expression of genes associated with fibrosis. (C) MyMRKO or MR antagonist ( $1 \mu$ M RU26752) alteration of LPS response. (D) Increases in $\mathrm{AM} \phi$ markers with IL-4 stimulation. ${ }^{\#} P<0.035,{ }^{\dagger} P<0.031$, Benjamini-Hochberg correction; ${ }^{*} P<0.05$, ${ }^{* *} P<0.01$ by 2 -tailed Student's $t$ test for multiple gene comparisons corrected for false discovery rate or 2-way ANOVA with Bonferroni post-tests.

pus (20). When cultured in normal serum and stimulated with LPS, eplerenone suppressed M1 markers, suggesting that MR is occupied and stimulatory when normal serum is present (Figure 1D).

However, when we tested this hypothesis in C/D medium, we found a proinflammatory effect of corticosterone at low concentrations, but this effect was blocked by a GR antagonist and not by an MR antagonist, consistent with previous reports (Figure 1E) (21). This raised the possibility that eplerenone was having an inverse agonist effect, hence suppressing the effect of MR below baseline. However, in C/D medium, eplerenone had no effect on baseline expression of Tnfa and did not block effects below baseline (Figure 1, A and E), which is consistent with occupation by an endogenous steroid in the presence of steroid.
Since classical and alternative activation have been reported to be competing pathways, we determined the effect on AM $\phi$ markers, which showed a stimulation of the majority of the markers tested (Figure 1F). Because of dramatic effects on fibrosis by aldosterone in vivo and the role of macrophages in fibrosis, we also tested the changes in expression of genes involved in fibrosis (Figure 1G). These included the profibrotic Tgfb and plasminogen activator inhibitor-1 (Pai1), with Pail being significantly suppressed. An inhibitor of TGF- $\beta$, Htra1, was significantly increased, as were two other genes associated with fibrosis, Pdk4 and Cdh2.

To better understand and test the role of MR in myeloid cells, we inactivated the $M R$ gene in a myeloid-specific knockout (MyMRKO). This knockout was produced by crossing floxed 
A

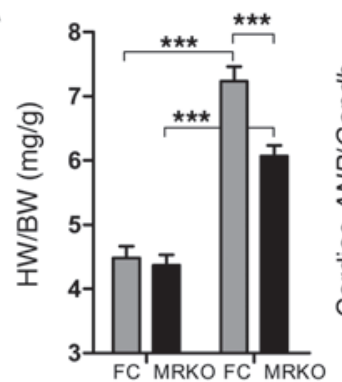

(-) L-NAME/Ang II

2-way ANOVA:

$P<0.01$ for MRKO $P<0.001$ for L-NAME/Ang ॥ $P<0.05$ for interaction

B

$\mathrm{H} \& \mathrm{E}$

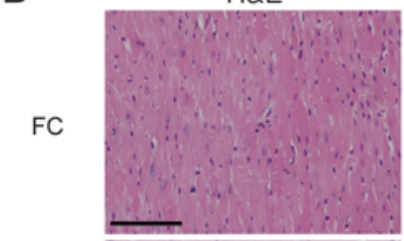

MRKO
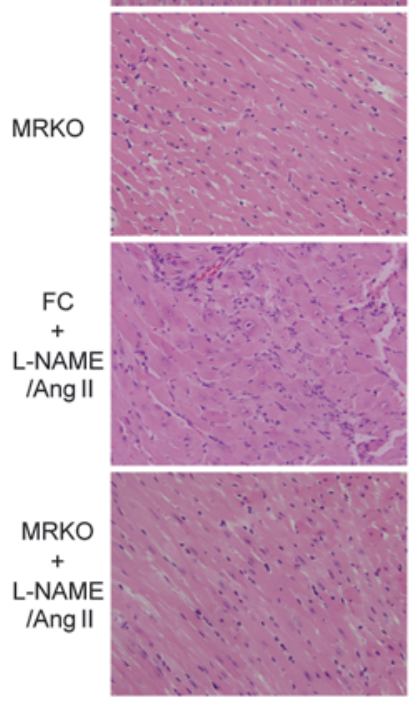

D

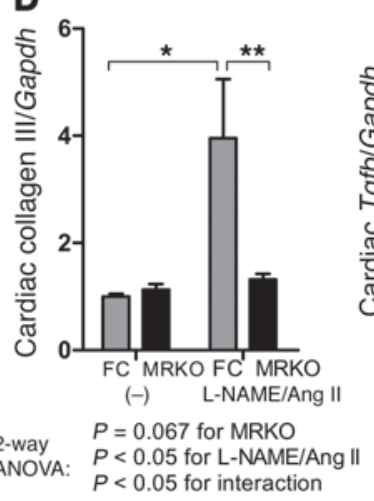

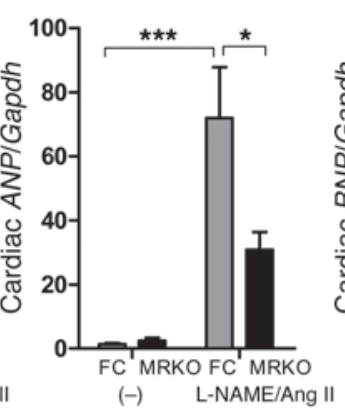

$P=0.058$ for MRKO

$P<0.001$ for L-NAME/Ang ॥

$P<0.05$ for interaction

Picrosirius red
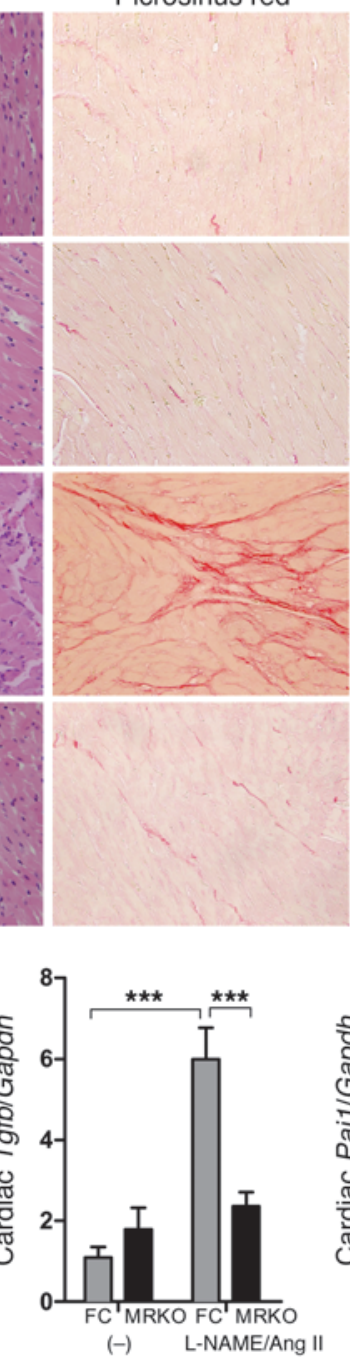

$P<0.05$ for MRKO

$P<0.001$ for L-NAME/Ang ॥

$P<0.01$ for interaction

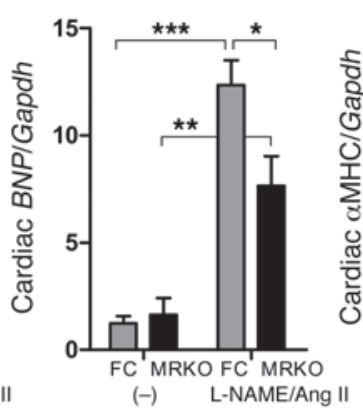

$P=0.088$ for MRKO

$P<0.001$ for L-NAME/Ang $\|$

$P<0.05$ for interaction

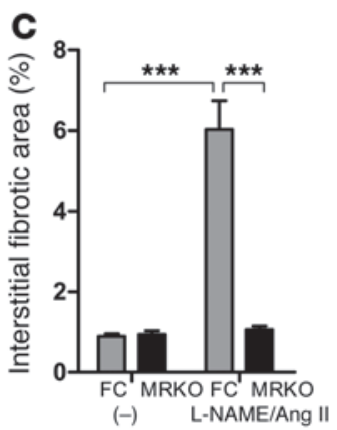

2-way $\quad P<0.001$ for MRKO

ANOVA: $P<0.001$ for L-NAME/Ang I

$P<0.001$ for interaction

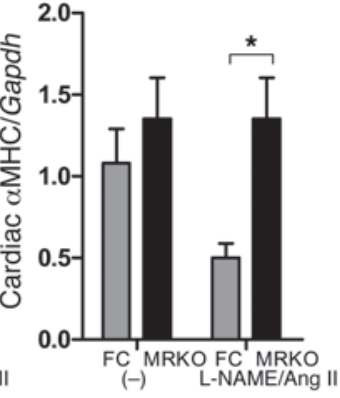

$P<0.05$ for MRKO

$P=0.19$ for $L-N A M E / A n g \|$

$P=0.19$ for interaction

\section{Figure 3}

MyMRKO protects against L-NAME/Ang II-induced cardiac hypertrophy and fibrosis. (A) Heart weight to body weight ratios and gene expression of markers of cardiac hypertrophy in FC and MyMRKO mice with or without (-) L-NAME/Ang II treatment (see Methods). (B) Representative H\&E and picrosirius red staining of cross sections of heart samples. Fibrotic tissues stained red. Scale bar: $100 \mu \mathrm{m}$. (C) Quantification of interstitial fibrotic areas. (D) Gene expression of markers of cardiac fibrosis. ${ }^{\star} P<0.05$, ${ }^{\star \star} P<0.01$, ${ }^{\star * \star} P<0.001$ by 2 -way ANOVA Bonferroni post-tests. 
$M R$ allele (22) with LysM-Cre (23) and resulted in the near complete inactivation of the gene in isolated PEMs (Supplemental Figure 1; supplemental material available online with this article; doi:10.1172/JCI41080DS1). We then used quantitative PCR (QPCR) to determine the expression changes associated with classical (M1) and alternatively activated macrophages $(\mathrm{AM} \phi)$. Similar to the in vitro response to MR antagonists, PEMs showed a reduction in most of the M1 markers and an increase in many $\mathrm{AM} \phi$ markers (Figure 2A). Most notably, expression of iNOS and Cd36 was not altered.

Also similar to the in vitro effects of MR antagonists, the knockout of MRcaused parallel changes in genes associated with fibrosis(Figure 2B). A comparison of mRNA levels between MRKO and floxed control (FC) macrophages using Affymetrix expression chips demonstrated that $\mathrm{MR}$ regulates multiple macrophage functions linked to wounding responses, including extracellular matrix structure, BMP signaling, and redox control (Supplemental Figure 2), consistent with a broad range of changes involved in tissue modeling.

When stimulated with LPS, MR antagonists inhibited the M1 response as before in WT cells but not in MRKO PEMs (Figure 2C). These results show that the effects of MR antagonists are MR dependent and not due to an off-target effect. MRKO PEMs showed reduced response for some but not all genes, suggesting that there was some compensation with the knockout compared with acute inhibition by MR antagonists.

IL-4, an inducer of one type of AM $\phi$, synergized with MRKO to display enhanced expression of AM $\phi$ markers (Figure 2D). This result may indicate that the MRKO macrophages are primed to polarize in the alternative activation direction, as was recently reported for stimulation of PPAR $\gamma$ (13). Interestingly, we demonstrate a decrease in Htra1 expression with IL-4 that is counteracted in the MRKO (Figure 2D). This result illustrates that there are important differences between the AM $\phi$ phenotype induced by MR inhibition and that induced by IL-4. We also determined the responses in resident peritoneal macrophages (Supplemental Figure 3). These macrophages had 30\%-40\% remaining $M R$ mRNA expression, indicating incomplete knockout. Since this was after isolation and culture, these results are comparable to the incomplete knockout of resident macrophage lineage cells in the brain, which do not express LysM in the quiescent state (24). Responses to LPS were largely intact (Supplemental Figure 3A), but there was an increase in the responsiveness to IL-4 for some AM $\phi$ genes (Supplemental Figure 3B).

Myeloid MRKO is protective against cardiac bypertrophy, inflammation, and fibrosis induced by L-NAME/Ang II. To test the hypothesis that MyMRKO would phenocopy the effects of MR antagonists in this in vivo model, we treated MyMRKO and control mice with a combination of a NOS inhibitor, $N^{\mathrm{G}}$-nitro-L-arginine methyl ester (L-NAME), and Ang II to generate cardiac and vascular damage and fibrosis (25). The cardiovascular injury in this model is mitigated by treatment with MR antagonists (26). Plasma corticosterone and aldosterone levels were similar in control and MyMRKO animals (Supplemental Table 1).

Cardiac hypertrophy was significantly less pronounced in the MyMRKO (Figure 3A). Importantly, there was also inhibition of the fetal gene program (increases in $A N P$ and $B N P$ and downregulation of $\alpha-M H C$; Figure $3 \mathrm{~A}$ ), indicating an important role for myeloid cells in modulating the cardiomyocyte responses and gene expression.

The MyMRKO mice showed dramatically less interstitial fibrosis, measured by picrosirius staining, which could have also contributed to the decreased heart size (Figure 3, B and C). This is essentially identical to the reported effects of eplerenone in this system (26). Expression of genes important in fibrosis, such as collagen III, Tgfb, and Pai1, was coordinately suppressed by MyMRKO compared with controls in the treated groups (Figure 3D).

Inflammatory infiltration of macrophages was also suppressed, as revealed by histological staining for the macrophage marker Mac2 (Figure 4A) and quantified in Figure 4B. This result indicates an alteration in myeloid trafficking and is corroborated by the decrease in the expression of the macrophage marker F4/80 (Figure 4C). Markers of M1 macrophages were suppressed (Tnfa, RANTES), whereas markers of AM $\phi$ (Fizz1, F13a1) were increased (Figure 4D) in MyMRKO relative to L-NAME/Ang II-treated FC mice. Similar findings are shown for perivascular inflammation and fibrosis (Figure 5).

$M y M R K O$ reduces vascular bypertrophy, inflammation, and fibrosis. L-NAME/Ang II-induced aortic remodeling, including fibrosis, decreased in the MyMRKO compared with treated FC mice. Picrosirius staining showed decreased fibrotic area in the treated MyMRKO mice compared with controls (Figure 6, A and B). The fibrotic area in treated controls appeared to be more diffuse within the expanded adventitia. Expression of the fibrosis-associated genes collagen III, Tgfb, and Pail was suppressed in treated MyMRKO mice (Figure 6C). Similarly, aortic wall thickness was decreased in treated MyMRKO animals (Figure 6D).

There was marked increase in macrophage staining in aortas from treated FC mice but much less from MyMRKO mice (Figure 7, $A$ and $B$ ). The increase was largely seen in the adventitia, but there was also some infiltration in the media and intima. The MyMRKO mice exhibited decreased expression of a marker for total $(F 4 / 80)$ (Figure 7C) and classically activated macrophages (Tnfa) and increased expression of a marker for alternative activation (Arg1) in MyMRKO compared with treated controls (Figure 7D).

These results demonstrate that MR directs macrophage polarization state by promoting classical macrophage activation and repressing alternative activation in a model of cardiac injury. MyMRKO causes a robust AM $\phi$ phenotype and leads to a decrease in the trafficking of the macrophages into the tissue and a marked reduction in M1 cytokines. The AM $\phi$ shift caused by MyMRKO correlates with protection in cardiac and vascular hypertrophy and inflammation.

Blood pressure response in MyMRKO mice. While the protection by eplerenone in this experimental model is reported to be independent of blood pressure lowering (25), hypertension is well established as a stimulus for cardiac and vascular hypertrophy and remodeling. It was therefore important to determine whether the MyMRKO mice had altered blood pressure. Since blood pressure has a diurnal rhythm and is affected by measurement conditions, we used continuous telemetry monitoring to assess unencumbered blood pressure throughout the day.

At baseline, the MyMRKO animals had no differences in blood pressure compared with FC animals (Figure 8). When the diet was changed to a high-salt diet, there was a small but significant increase in light-period blood pressure in the MyMRKO animals. Addition of L-NAME further increased the difference and particular led to a decrease in the magnitude of the circadian rhythm. These changes in circadian rhythm were most pronounced in the pulse pressure.

When Ang II was added, blood pressure differences between knockout and control mice were reduced, but pressures were still slightly higher in the MyMRKO mice. The most striking finding was that MyMRKO mice showed increased pulse pressure and a near complete loss of pulse pressure circadian variation. 
A
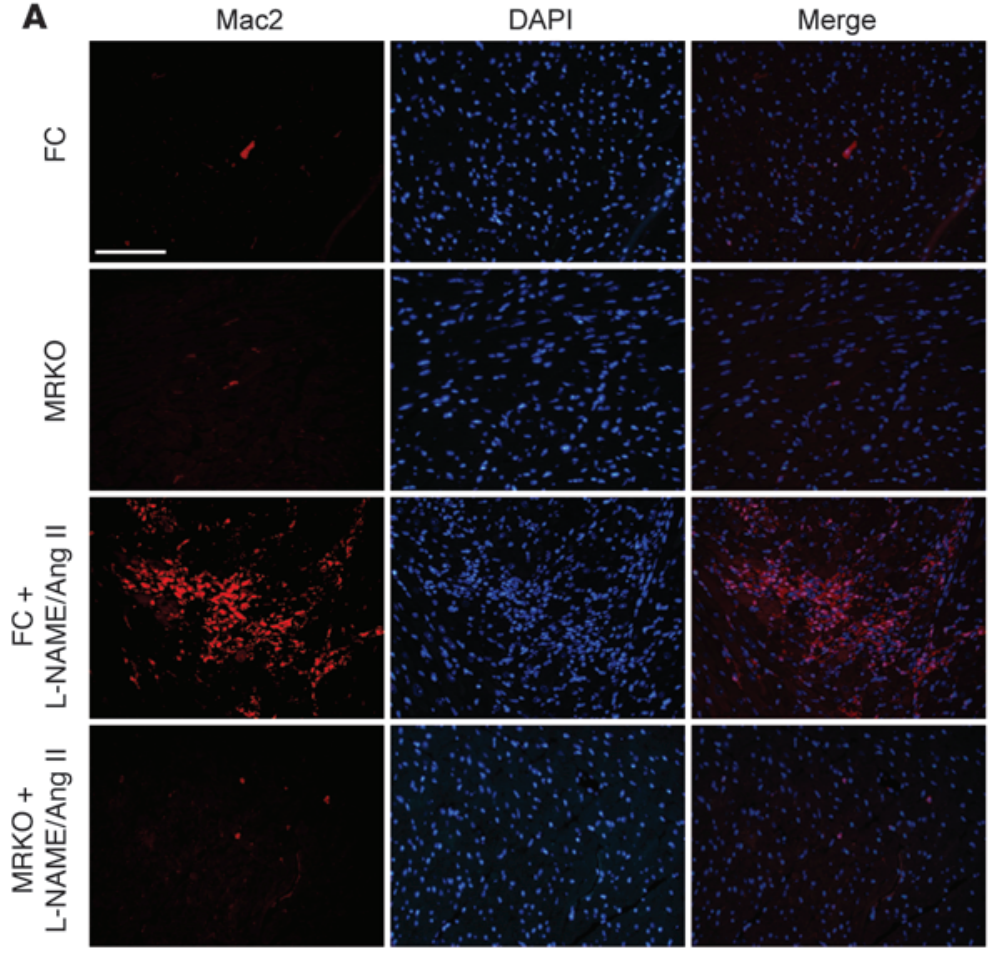

BF

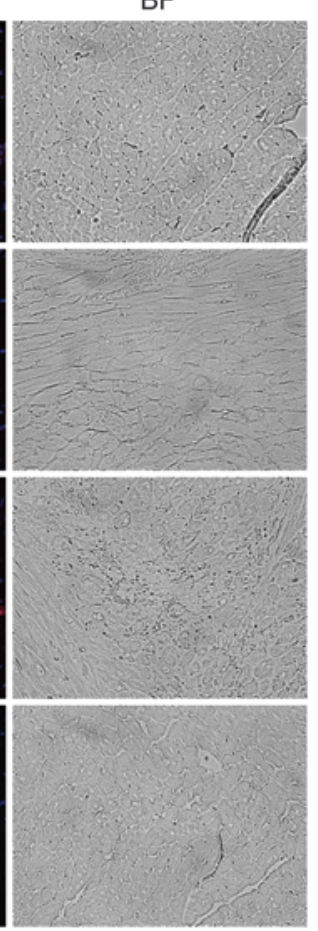

B

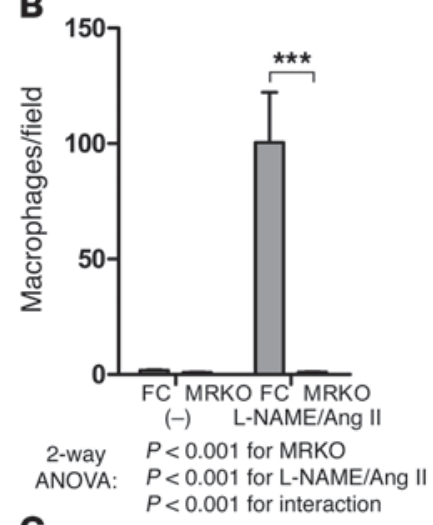

C

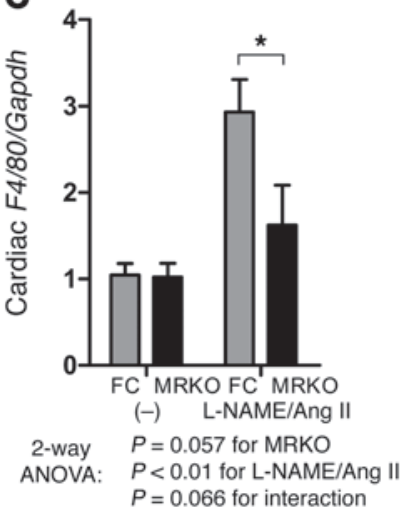

D

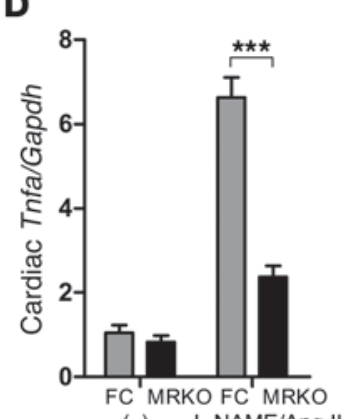

$(-) \quad$ L-NAME/Ang ॥

2-way $\quad P<0.001$ for MRKO

ANOVA: $\quad P<0.001$ for L-NAME/Ang II

$P<0.001$ for interaction

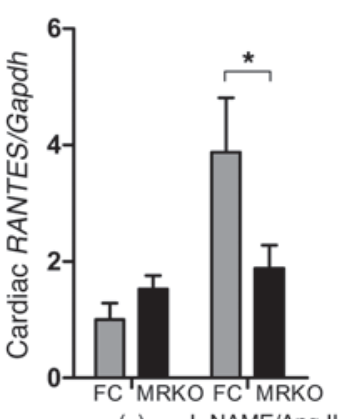

$(-) \quad$ L-NAME/Ang ॥

$P=0.2$ for MRKO

$P<0.05$ for L-NAME/Ang II

$P<0.05$ for interaction

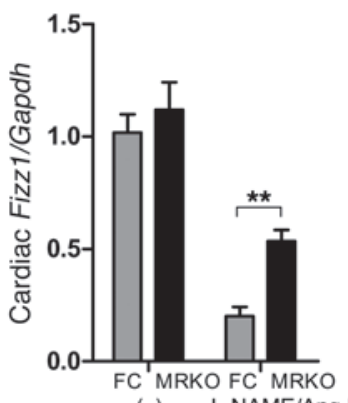

$(-) \quad$ L-NAME/Ang ॥ $P<0.01$ for MRKO

$P<0.001$ for L-NAME/Ang II

$P=0.12$ for interaction

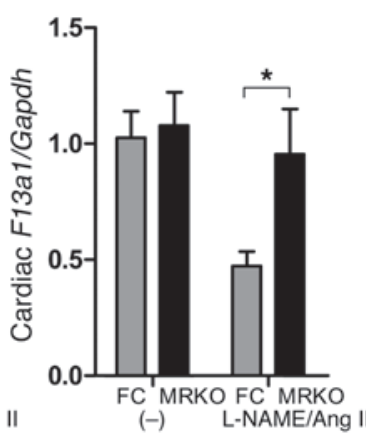

$P=0.077$ for MRKO

$P<0.05$ for L-NAME/Ang II

$P=0.15$ for interaction

\section{Figure 4}

MyMRKO reduces L-NAME/Ang II-induced interstitial macrophage recruitment in heart. (A) Representative immunofluorescence assay of cross sections of heart samples from FC and MyMRKO mice with or without L-NAME/Ang II treatment. Mac2 antibody was used to detect macrophages. Scale bar: $100 \mu \mathrm{m}$. BF, bright field. (B) Quantification of interstitial macrophages stained by Mac2. The results are expressed as macrophage counts per high-power field $(\times 400)$ under microscope. (C) Gene expression of macrophage marker F4/80. (D) Gene expression of M1 markers (Tnfa and RANTES) and AM $\Phi$ markers (Fizz1 and F13a1). ${ }^{*} P<0.05,{ }^{* \star} P<0.01$, ${ }^{* * *} P<0.001$ by 2-way ANOVA Bonferroni post-tests.

Interaction of $M R$ with PPAR . The effects of MR antagonism and MRKO are similar to those of the thiazolidinediones (TZDs), PPAR $\gamma$ agonists, in their ability to mitigate cardiovascular inflammation and fibrosis through their action on macrophages $(18,27)$. TZDs also enhance some aspects of alternative activation in macrophages, suggesting a potential common mechanism $(13,28)$. In macrophages from myeloid PPAR $\gamma$-knockout (MyPGKO) mice, the changes in expression of many of the genes associated with macrophage polarization were opposite those in MyMRKO macrophages, as assessed by QPCR (Figure 9A). Although MR antagonists have previously been shown to regulate PPAR $\gamma$ in some systems (6), MR deletion did not affect PPAR $\gamma$ expression. However, since effects were similar, we could not rule out alteration in PPAR $\gamma$ activity. PPAR $\gamma$ deletion did increase MR expression, raising the possibility that changes in MR expression contributed to the proinflammatory phenotype. We therefore chose several markers to determine whether PPAR $\gamma$ and MR acted upstream or downstream of each other or whether they altered macrophage polarization by parallel pathways. We tested the action of MR antagonists in PPAR $\gamma$-null macrophages and the PPAR $\gamma$ agonist pioglitazone in MR-null macrophages (Figure 9, B and C). 
A

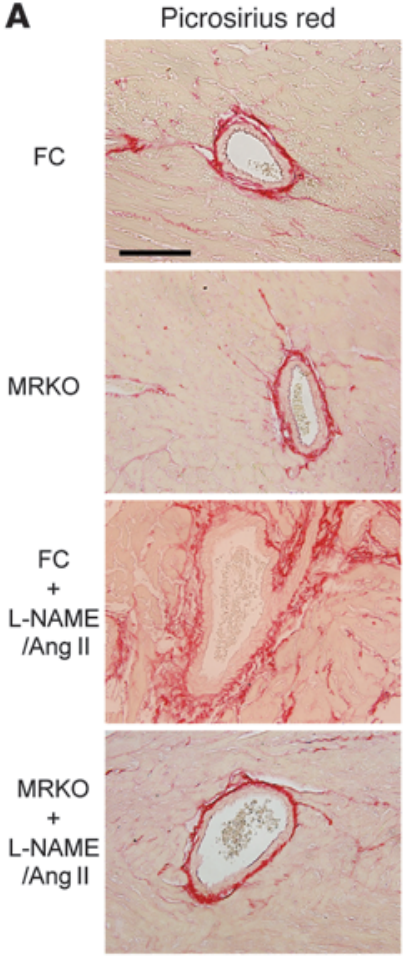

B

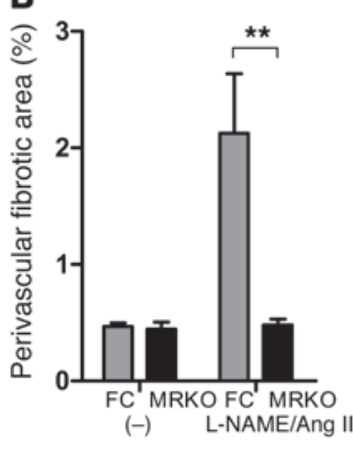

2-way $P<0.01$ for MRKO

ANOVA: $P<0.01$ for L-NAME/Ang II

$P<0.01$ for interaction

Mac2
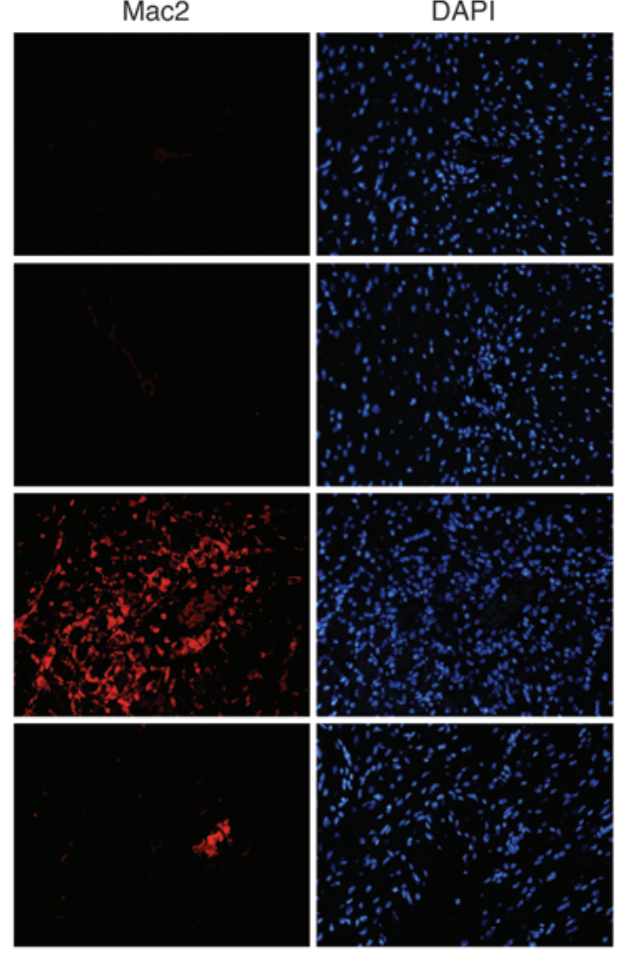

C
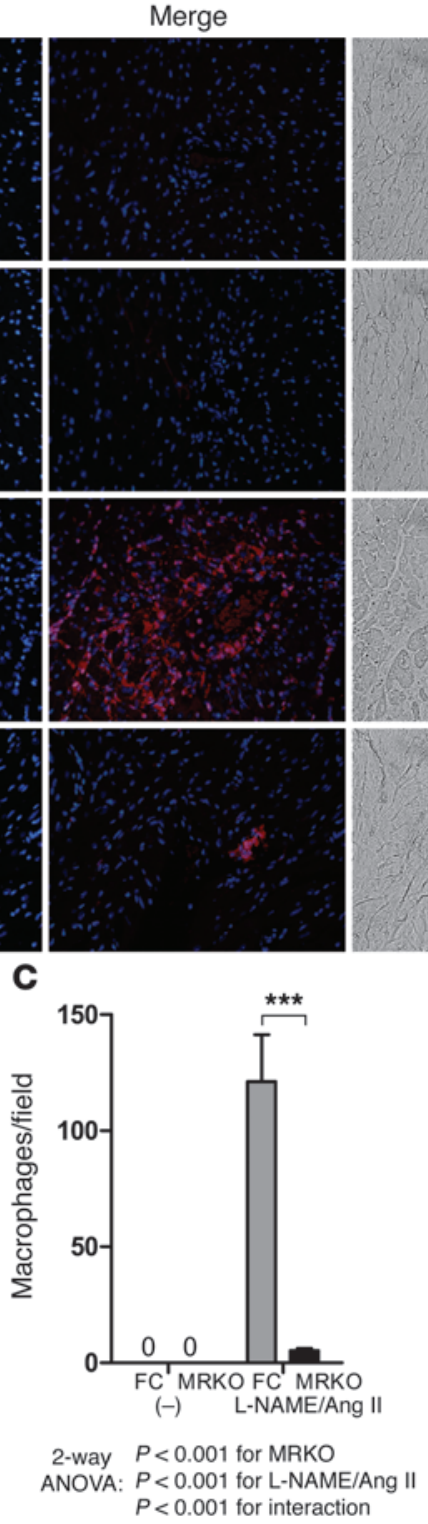

\section{Figure 5}

MyMRKO reduces perivascular fibrosis and macrophage recruitment induced by L-NAME/Ang II in heart. (A) Representative picrosirius red staining and immunofluorescence assay of cross sections of heart samples from FC and MyMRKO mice with or without L-NAME/Ang II treatment. Fibrotic tissues stained red. Mac2 antibody was used to detect macrophages. Scale bar: $100 \mu \mathrm{m}$. (B) Quantification of perivascular fibrotic areas. (C) Quantification of perivascular macrophages stained by Mac2. The results are expressed as macrophage counts per high-power field (×400) under microscope. ${ }^{* *} P<0.001$ by 2 -way ANOVA Bonferroni post-tests.

PPAR $\gamma$ agonists were effective in MR-null cells. In fact, MRKO and TZD can be synergistic. Some genes such as Ym1 that showed a poor response to pioglitazone in control cells showed a markedly enhanced response in MRKO cells. Conversely, MR agonists were effective in PPAR $\gamma$-null cells at inducing the M1 marker TNF- $\alpha$ and suppressing the AM $\phi$ marker Arg1 (Figure 9C). Similarly, opposing actions on IL-4 induced expression of Ccl7 were seen (Figure 9D) These results show that they act by parallel but interacting pathways and that modification of multiple pathways may give enhanced responses.

Interaction of MR with GR. MRKO has an effect similar to that of GR agonists in that they both produce a phenotype resembling IL-4- induced M2 polarization (9). Therefore, we investigated whether MRKO could modulate the GR response, including induction of the AM $\phi$ phenotype. Since a majority of MR's evolution occurred prior to the existence of physiologic aldosterone, this cellular role is likely a conserved ancestral function, observable in cell types such as neurons, cardiomyocytes, and macrophages $(29,30)$. Transcriptional changes caused by MRKO overlapped with the glucocorticoid response (Figure 10A and Supplemental Figures 4 and 5). However, the majority of genes regulated by glucocorticoids were unaffected by MRKO, whereas about half of the MRKO-altered genes were also regulated by glucocorticoids. By cluster analysis, we separated the different responses to corticosterone in FC and 
A

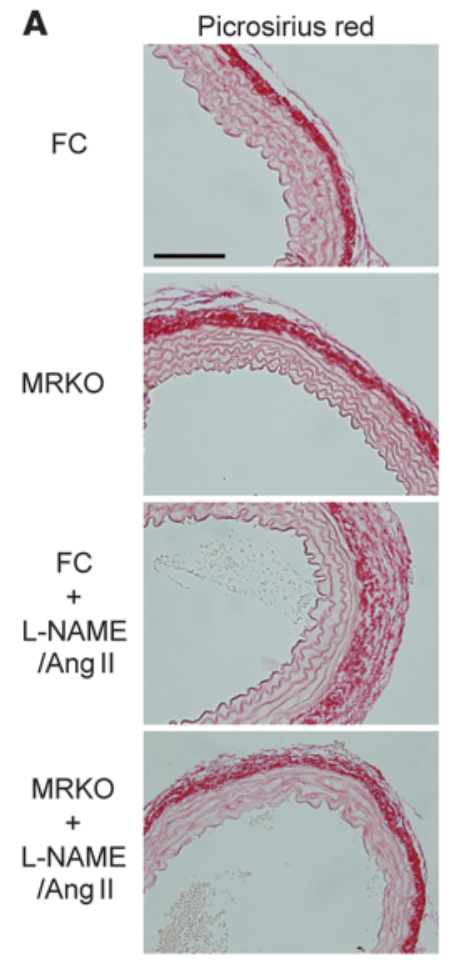

B

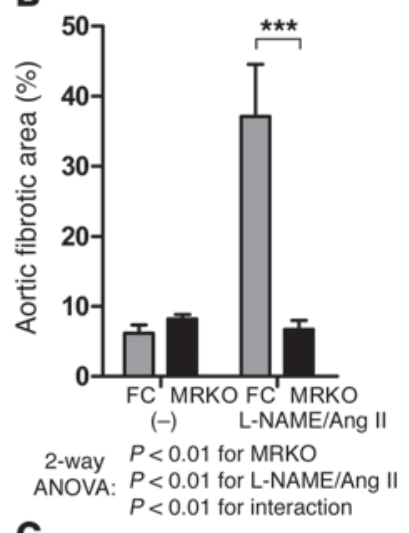

C

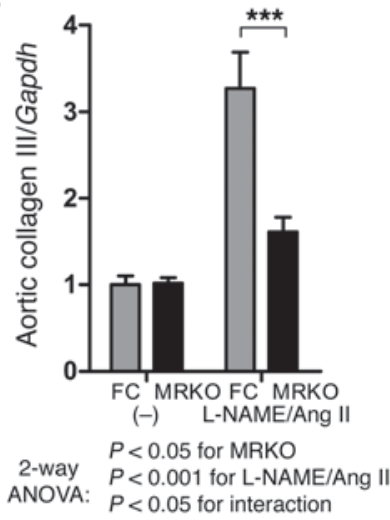

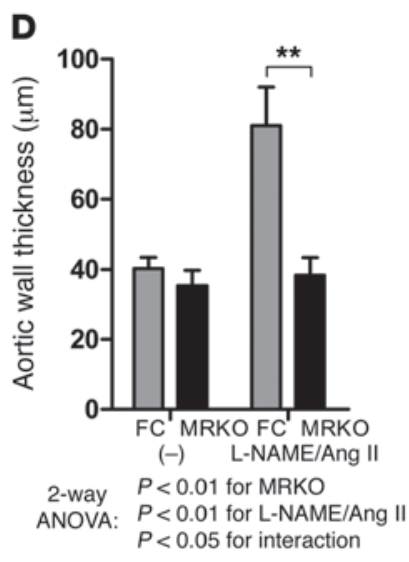

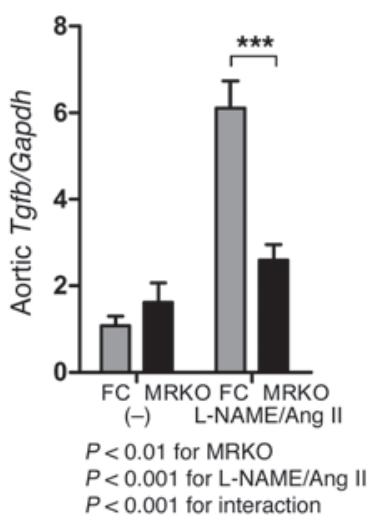

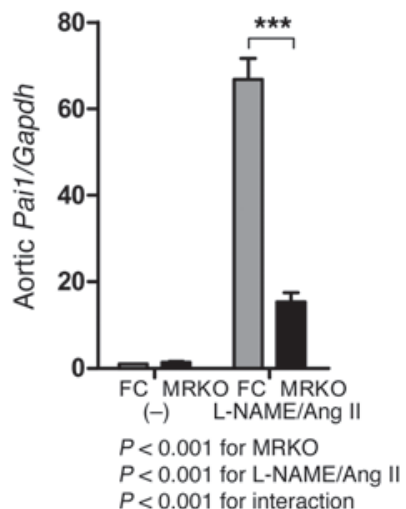

\section{Figure 6}

MyMRKO protects against L-NAME/Ang II-induced aortic fibrosis and hypertrophy. (A) Representative picrosirius red staining of cross sections of aortas from FC and MyMRKO mice with or without L-NAME/Ang II treatment. Fibrotic tissues stained red. Scale bar: $100 \mu \mathrm{m}$. (B) Quantification of aortic fibrotic areas. (C) Gene expression of markers of cardiac fibrosis. (D) Quantification of aortic hypertrophy. ${ }^{\star \star} P<0.01,{ }^{* \star *} P<0.001$ by 2-way ANOVA Bonferroni post-tests.

MyMRKO macrophages according to their correspondence to genes where MRKO mimicked or enhanced the response to corticosterone (cluster A); genes where MRKO and corticosterone caused suppression of expression (cluster B); and genes where corticosterone opposed the inductions caused by MRKO (cluster C) (Figure 10B). Cluster A contained many AM $\phi$ markers. The synergy between MRKO and corticosterone in inducing enhanced expression of AM $\phi$ markers $Y m 1$ and $F 13 a 1$ was confirmed by QPCR (Figure 10C). Also in cluster A are the proteases Htra1 (a TGF- $\beta$ inhibitor) and Serpine2, which showed marked synergy and is a relative of Pail but has not been linked to cardiovascular disease (Figure 10C). Cluster B included genes where MRKO and corticosterone synergized to repress the expression (Cdh1, IL27a) or where MRKO and corticosterone in FC cells suppressed the expression but addition of corticosterone to the MRKO cells did not result in further suppression (Il1b and Clec2, a proinflammatory prothrombotic C-type lectin) (Figure 10D). Cluster C had a number of different genes - including formyl-peptide receptor 2 , involved in chemotaxis, and adrenomedullin $(A d m)$, which has been linked to atherosclerosis protection - that are involved in recruitment of macrophages and may contribute to the protective effects of the knockout.

Cluster analysis of M1- and AM $\phi$-related genes evaluated by QPCR for the 4 treatments clearly separated the two groups. It also showed that there were marked differences in the AM $\phi$-associated genes between the different treatments and separated the genes into those that are in agreement (which contained the most often used $\mathrm{AM} \phi$ markers) and genes where there are marked differences (Figure 11A). Consistent with the previously described changes, M1 markers were increased by PGKO and decreased by MRKO, corticosterone, and IL-4. Opposite effects were seen with the commonly used AM $\phi$ marker Arg1. Other AM $\phi$ markers, such as $Y m 1$ and Fizz1, showed concordance between corticosterone, MRKO, and IL-4, although the magnitude of changes could be very different. Other genes showed marked differences and point out that the $\mathrm{AM} \phi$ are different depending on the treatment. Some marked differences were in the cadherins, where MRKO and corticosterone had effects opposite to IL-4. Therefore, there is a system of opposing and cooperating nuclear transcription factors that are capable of modifying the polarization phenotype of macrophages, thus modifying the response to injury and disease in the cardiovascular system. Further delineation of the phenotype similarities and differences of these subtypes may reveal the particular function of this spectrum of macrophage polarization (Figure 11B).

\section{Discussion}

These results delineate for the first time to our knowledge an important role of myeloid cells in controlling cardiac hypertrophy and vascular changes in response to L-NAME/Ang II. Importantly, our data demonstrate that the effect of L-NAME/Ang II on 
A
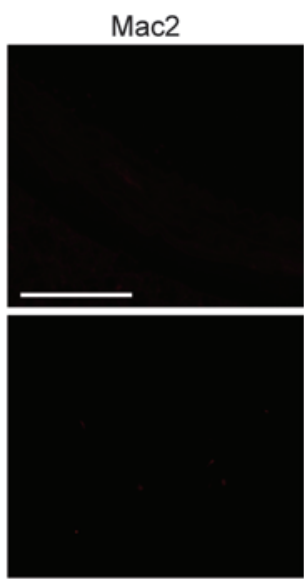

FC

$\stackrel{+}{+}$

IAng |I
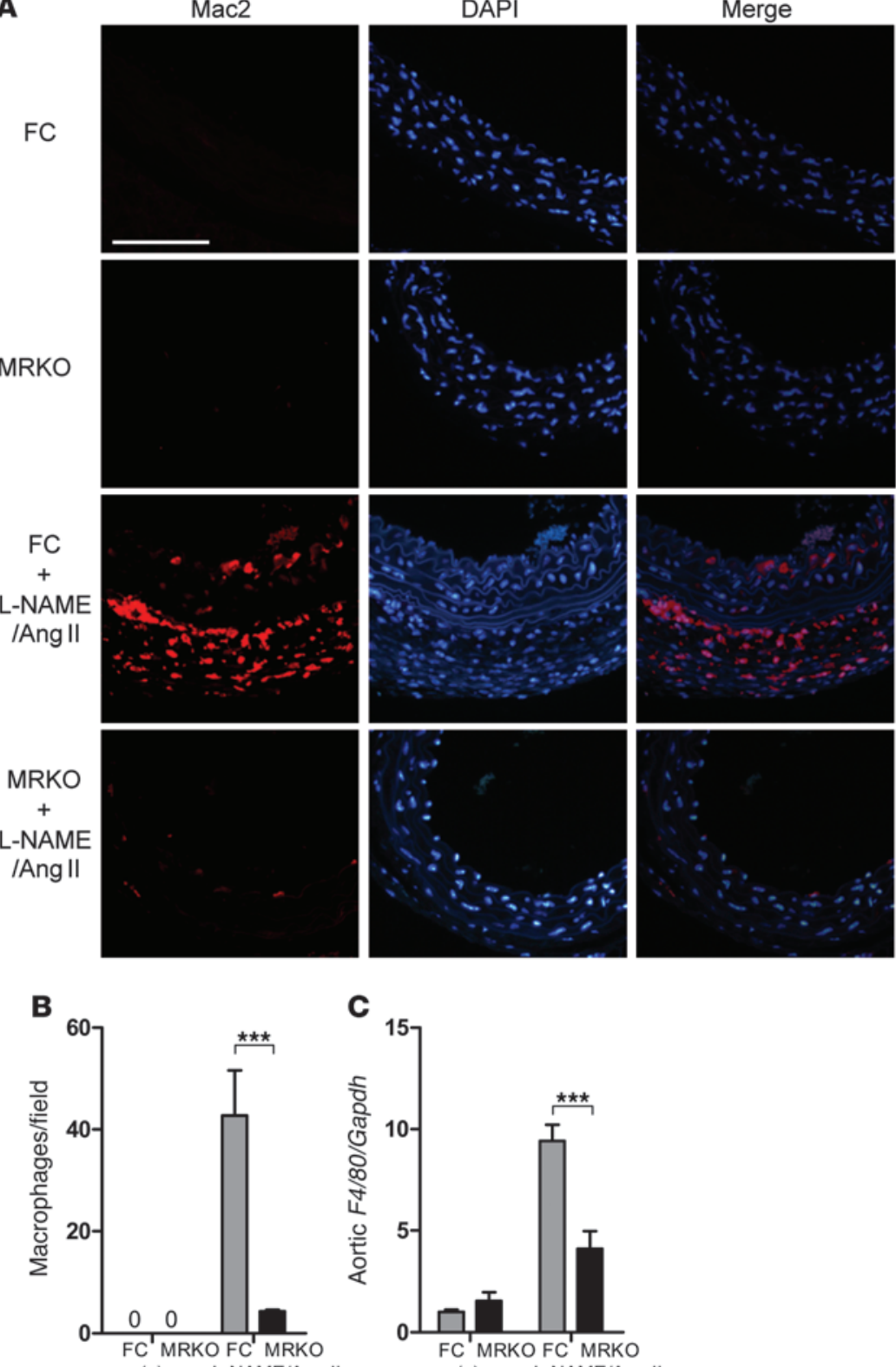

(-) L-NAME/Ang I

$$
\text { 2-way } P<0.01 \text { for MRKO }
$$

ANOVA: $P<0.001$ for L-NAME/Ang II

$P<0.001$ for interaction

D

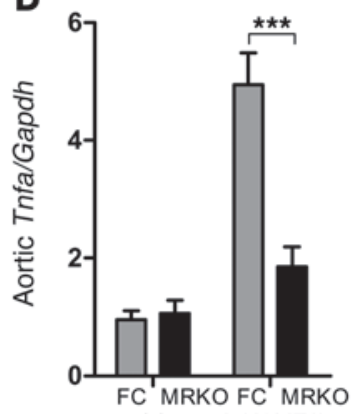

$(-) \quad$ L-NAME/Ang II

$P<0.01$ for MRKO

2-way $\quad P<0.001$ for L-NAME/Ang ॥

ANOVA: $P<0.001$ for interaction

\section{C}

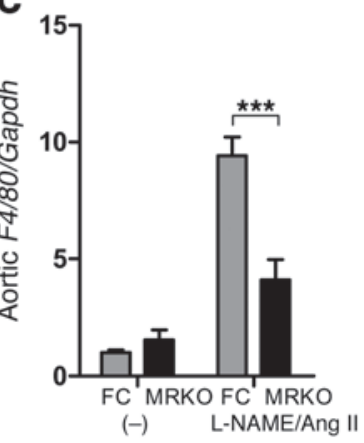

$P<0.01$ for MRKO

$P<0.001$ for L-NAME/Ang II

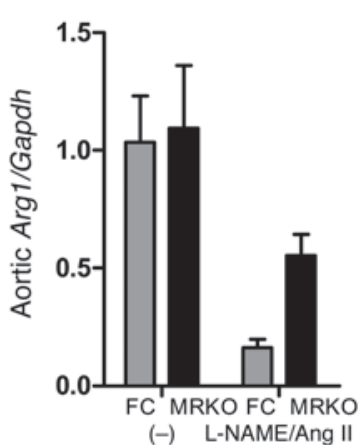

$P=0.17$ for MRKO

$P<0.001$ for L-NAME/Ang II

$P=0.31$ for interaction
$P<0.001$ for interaction

\section{Figure 7}

MyMRKO reduces L-NAME/Ang II-induced aortic macrophage recruitment. (A) Representative immunofluorescence assay of cross sections of aortas from FC and MyMRKO mice with or without L-NAME/Ang II treatment. Mac2 antibody was used to detect macrophages. Scale bar: $100 \mu \mathrm{m}$. (B) Quantification of aortic macrophages stained by Mac2. The results are expressed as macrophage counts per highpower field $(\times 400)$ under microscope. (C) Gene expression of macrophage marker F4/80. (D) Gene expression of M1 marker Tnfa and AM $\phi$ marker Arg 1. ${ }^{* * *} P<0.001$ by 2-way ANOVA Bonferroni post-tests. 

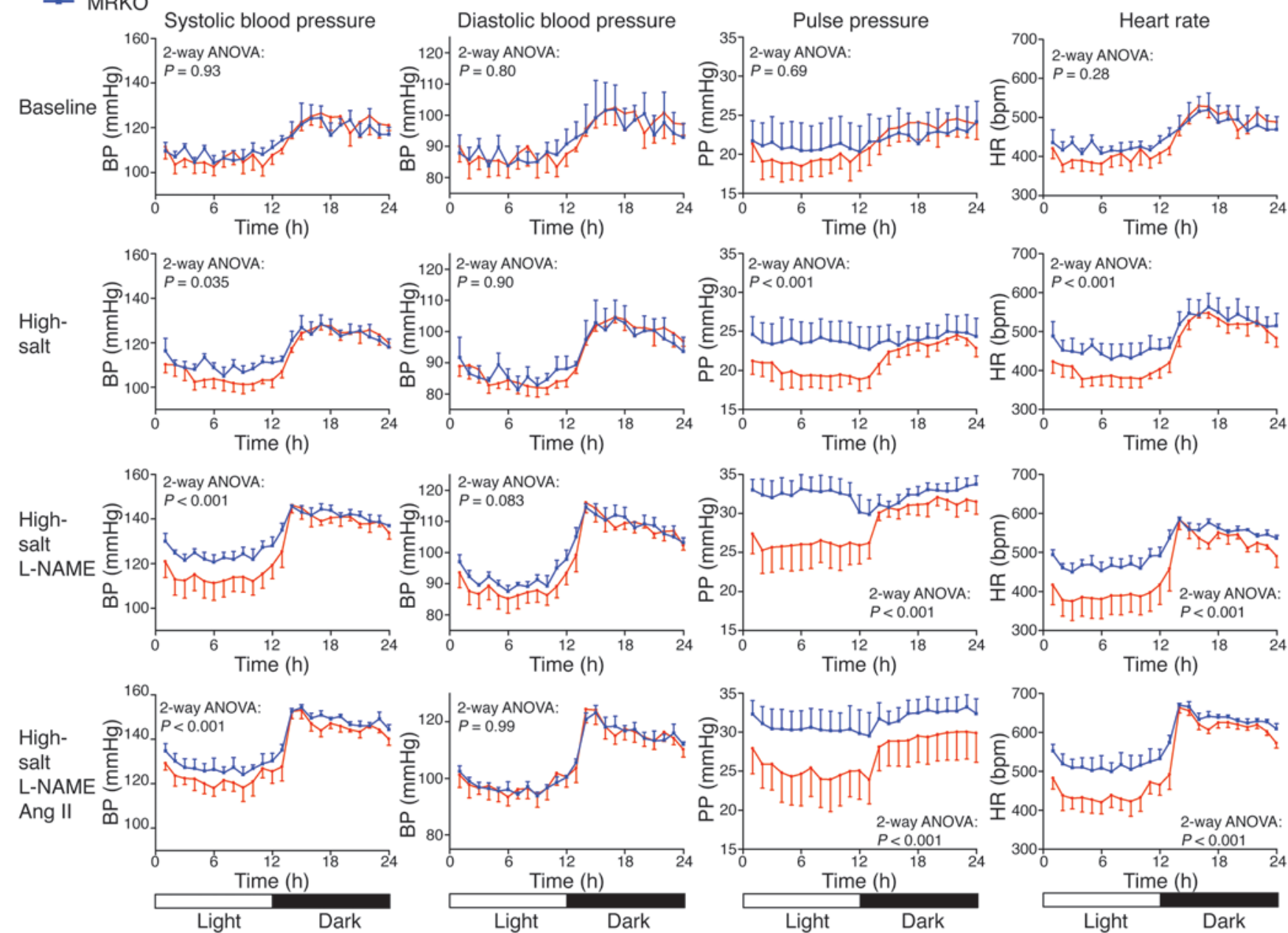

Figure 8

MyMRKO causes an increased blood pressure and heart rate response to L-NAME/Ang II. Twenty-four-hour averages of blood pressure and heart rate data collected by radiotelemetry under each condition of the model show a significant increase in daytime systolic pressure, pulse pressure, and heart rate in MyMRKO versus FC mice.

raising blood pressure is intact or even enhanced, showing that these improvements are not caused through lessening of blood pressure. Further, the data show that myeloid MR is critical in controlling the macrophage phenotype and that its absence leads to $\mathrm{AM} \phi$ polarization. In isolated macrophages, pharmacologic MR blockade has similar effects, thus demonstrating that these are direct effects on the macrophages. Macrophage activation polarization is important in the inflammation associated with obesity (31), so these results have implications far beyond these studies. It is possible that these changes may also represent a link between aldosterone and insulin resistance that has been seen with hyperaldosterone states (32).

This report differs from a previous report (33) of MRKO in DOCA/salt-treated animals, where the hypertension was blocked and apparently cardiac hypertrophy was still present. The differences seen in blood pressure response may be due to differences in the models or the technique used for measurement (tail cuff vs. direct measurement). The previous publication also did not report any statistically significant difference between DOCA/salt- treated control and DOCA/salt-treated knockout animals in macrophage recruitment, expression of macrophage markers, or genes involved in fibrotic responses or collagen content. Our data clearly show differences between L-NAME/Ang II-treated controls and MyMRKO mice in fibrosis and macrophage trafficking.

The ligand for the myeloid MR has been presumed to be a glucocorticoid, since natural glucocorticoids have the same affinity for the receptor as mineralocorticoids but circulate at 100-1,000 times the mineralocorticoid concentration, and the myeloid cells lack the glucocorticoid-inactivating enzyme $11 \beta \mathrm{HSD} 2$. These results partially support the idea that a normally circulating endogenous circulating steroid is proinflammatory in isolated macrophages. MR blockade is antiinflammatory in the presence of serum but not in the absence of steroids with charcoal/dextrantreated serum, which indicates that MR blockers are not acting as inverse agonists in macrophages in this system, as has been suggested in heart (34) - although given the experimental protocol, it was not clear whether the target was MR. Aldosterone at low concentrations (expected to occupy MR but not GR) is pro- 
A

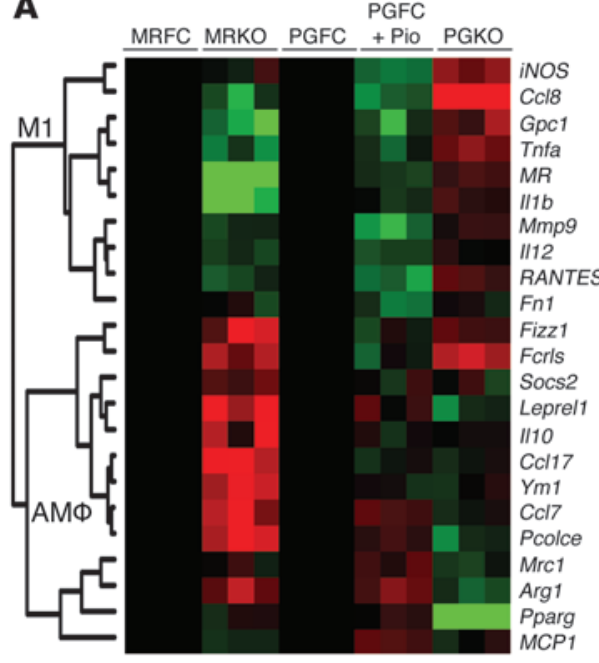

Fold change from untreated FC

$\begin{array}{lllllll}3 & 2.25 & 1.75 & 0 & 0.57 & 0.44 & 0.33\end{array}$
B
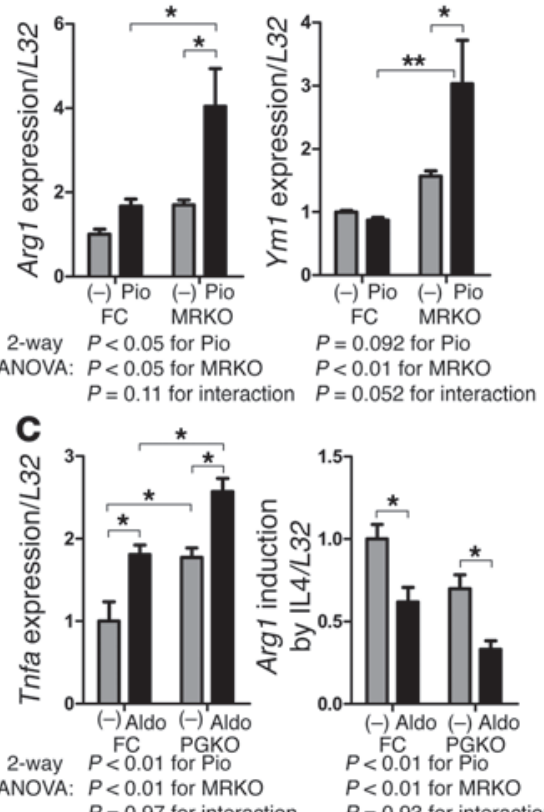

$P=0.97$ for interaction

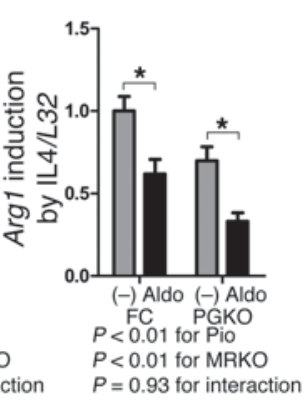

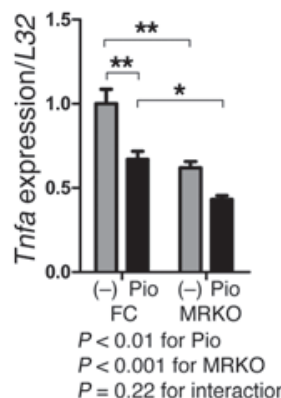
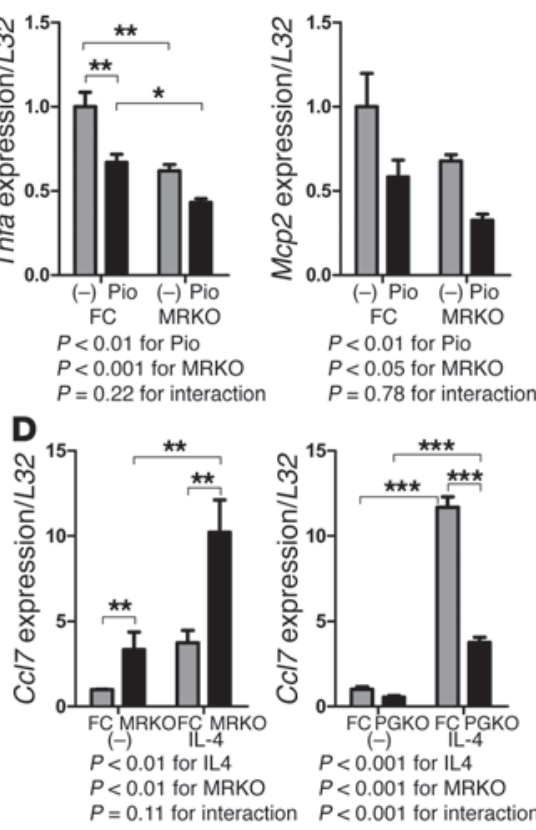

\section{Figure 9}

MR opposes PPAR $\gamma$ in macrophage polarization. (A) Hierarchical clustering of M1 and AM $\phi$ marker gene expression measured by real-time PCR from primary peritoneal macrophages demonstrates significant overlap between PPAR $\gamma$ activation and MyMRKO. (B) Deletion of MR enhanced the $A M \phi$-polarizing effects of $10 \mu \mathrm{M}$ pioglitazone (Pio). (C) MyPGKO macrophages cultured in C/D medium showed increases in M1 markers that were enhanced by addition of $10 \mathrm{nM}$ aldosterone. (D) MyMRKO and MyPGKO respectively enhance and oppose IL-4 stimulation of the AM $\phi$ marker Ccl7. MRFC, FC for MyMRKO; PGFC, FC for MyPGKO; PGKO, MyPGKO; Cort, corticosterone. ${ }^{*} P<0.05,{ }^{* \star} P<0.01$, ${ }^{* * *} P<0.001$ by 2 -way ANOVA Bonferroni post-tests.

inflammatory in charcoal/dextran-treated serum. In contrast, in the charcoal/dextran-treated serum, added low-dose glucocorticoids are proinflammatory but not through $\mathrm{MR}$, as the increase in TNF- $\alpha$ was blocked by GR antagonists and not MR antagonists as reported previously (21). While this may indicate that there is another occupying steroid, another possibility may be that the cells cultured differently have different properties. Culture with charcoal/dextran-treated serum had less proinflammatory activity, greater IL-10 secretion, and lower responses to LPS compared with culture with normal serum, suggesting an altered phenotype between the two cultures. Therefore, the nature of the cells cultured under these two conditions is different and may be responsible for the apparent contradictions.

MR and GR largely act in opposite directions in the determination of macrophage polarization. In addition, MR has long been known to coordinate the cellular response to glucocorticoids in tissues lacking $11 \beta \mathrm{HSD} 2$, such as the brain, where MR controls an overlapping counterregulatory program against the actions of GR $(35,36)$. In macrophages, MR and GR also have largely opposing roles, and the effects of MR are independent of GR.

Therefore, for most genes, MR and GR act by independent pathways resulting in a similar but distinct macrophage phenotype. These data suggest that, as in other tissues (37), MR and GR coordinate counterregulatory responses to changing glucocorticoid concentrations and that for a few responses, transcriptional repression caused by elevated glucocorticoid levels requires MR. Currently, it is unclear whether these interactions are direct or reflect an indirect action of the MRKO.
We conclude that MR evolved an important role in controlling classical and alternative macrophage activation likely representing a conserved ancestral corticosteroid receptor function. We have shown that macrophage MR deletion or inhibition eliminates a suppression of AM $\phi$ polarization, resulting in a macrophage phenotype that shares features of AM $\phi$ subtypes induced by IL-4, TZDs, or glucocorticoids (acting through GR), in addition to a coordinated reduction in classically activated macrophage markers. These responses were paired with an activation of a transcriptional program associated with ECM structure, bone morphogenetic protein (BMP) and growth hormone signaling, and redox control. This is consistent with the hypothesis that $\mathrm{AM} \phi$ activation is important in the structural and cellular response to wound healing.

Loss of macrophage MR activity exerts a protective effect on experimentally induced cardiac and vascular remodeling, largely mimicking the effects of MR antagonists. Therefore, a critical mechanism by which MR antagonists are cardioprotective is likely blocking of MR in macrophages, which would explain the benefit of MR antagonists in the absence of hyperaldosteronism.

In other cell types that lack $11 \beta$ HSD2 such as neurons, MR and GR play opposing cellular roles through independent but overlapping mechanisms $(35,36)$. We observed a similar role in macrophages, where MR and GR can drive opposite polarizing responses, largely through independent mechanisms. These nonredundant functions allow MRKO to induce multiple facets of AM $\phi$ polarization that are stimulated by glucocorticoids, PPAR $\gamma$, and IL-4. This interaction is likely important in MR's 
A
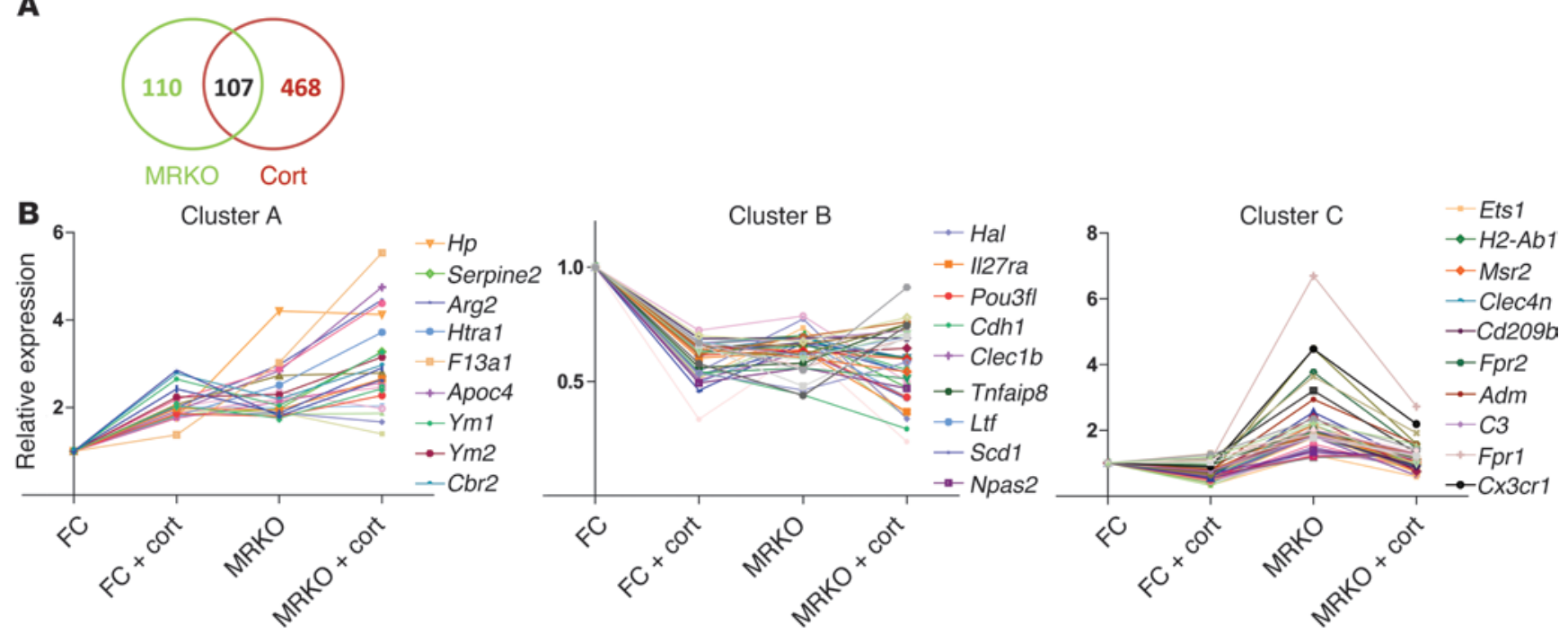

C
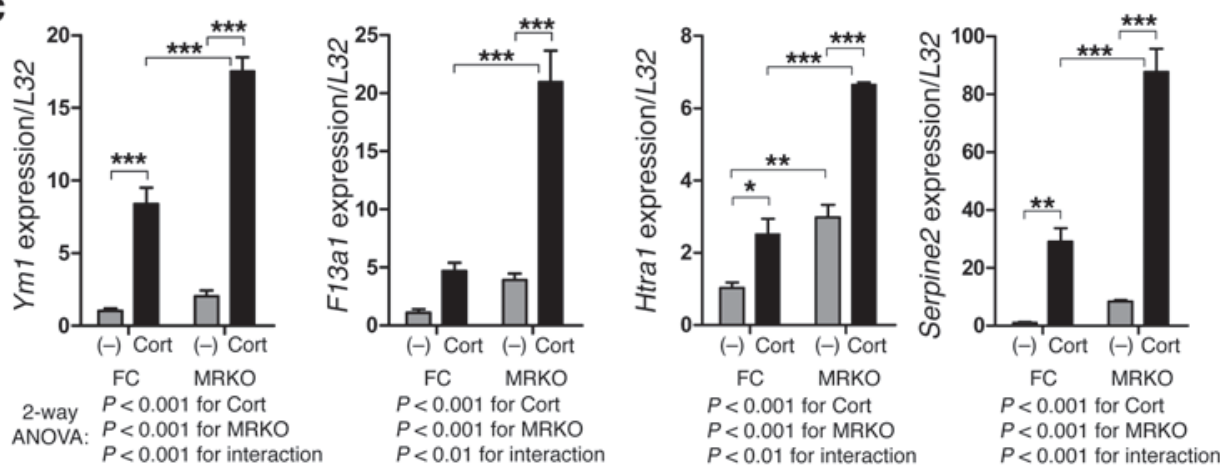

D
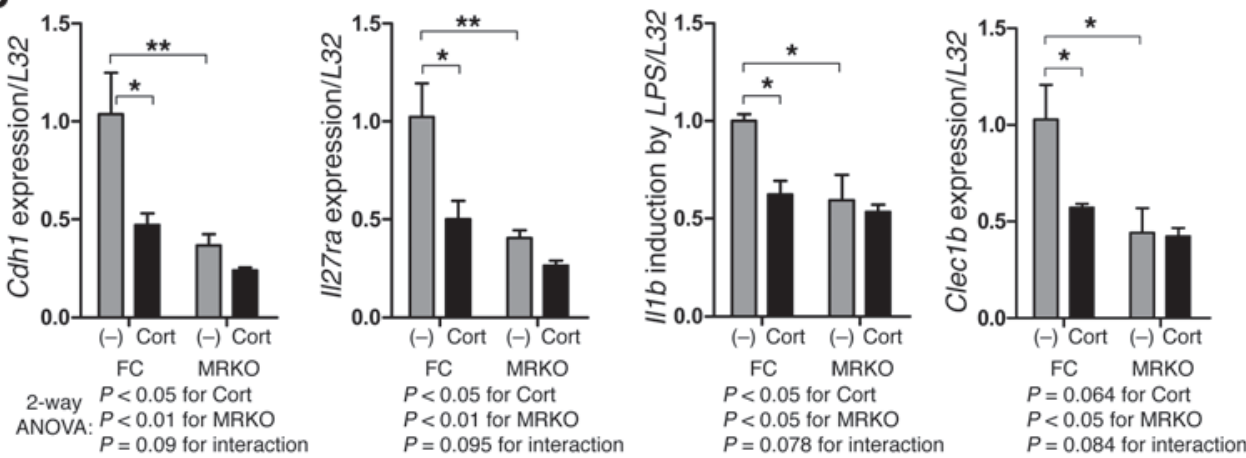

Figure 10

MR cooperates with glucocorticoid signaling in macrophages. (A) Affymetrix analysis of peritoneal macrophages treated with $1 \mu \mathrm{M}$ corticosterone for 24 hours shows a minority of genes regulated by MR and GR activation. (B) MyMRKO can enhance the induction of AM $\phi$ by corticosterone (cluster A), mimic the repression of proinflammatory factors by corticosterone (cluster B), or have the same effect as corticosterone but an not an additional effect with combination (cluster C). Representative genes are listed to the right of each cluster. (C) MyMRKO synergized with corticosterone to induce genes important in AM $\phi$ macrophage polarization (Ym1 and F13a1) and enhance the TGF- $\beta$ inhibitor Htra1 and thrombin inhibitor Serpine2. (D) MyMRKO synergized with corticosterone to repress TGF- $\beta$ target E-cadherin (Cdh1) and IL-27 receptor. In addition, MyMRKO abolished additional repression of $I / 1 \mathrm{~b}$ and the proinflammatory C-type lectin Clec2 by corticosterone. ${ }^{*} P<0.05,{ }^{* *} P<0.01$, ${ }^{\star * \star} P<0.001$ by 2 -way ANOVA Bonferroni post-tests.

role in enhancing cardiac injury and fibrosis, and the protective benefit conferred by MyMRKO or MR antagonism.

The differences in blood pressure between MyMRKO and control mice with Ang II and L-NAME treatment were most dramatic during the light period, and analysis of the pulse pressure shows a near loss of circadian rhythm. MyMRKO mice had higher blood pressure and heart rate compared with controls on high-salt diet, again, most significantly during the light period. Macrophages have recently been proposed as a factor in the control of salt sensitivity by modulating the lymphatic response (38). This loss of 


\section{A}

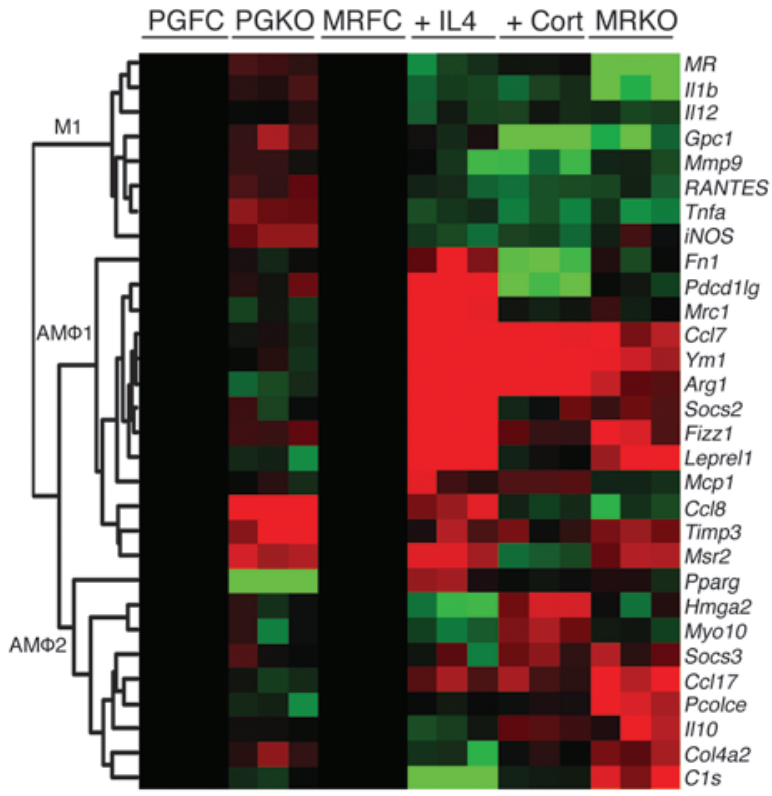

Fold change from untreated $\mathrm{FC}$

$\begin{array}{lllllll}3 & 2.25 & 1.75 & 0 & 0.57 & 0.44 & 0.33\end{array}$
B

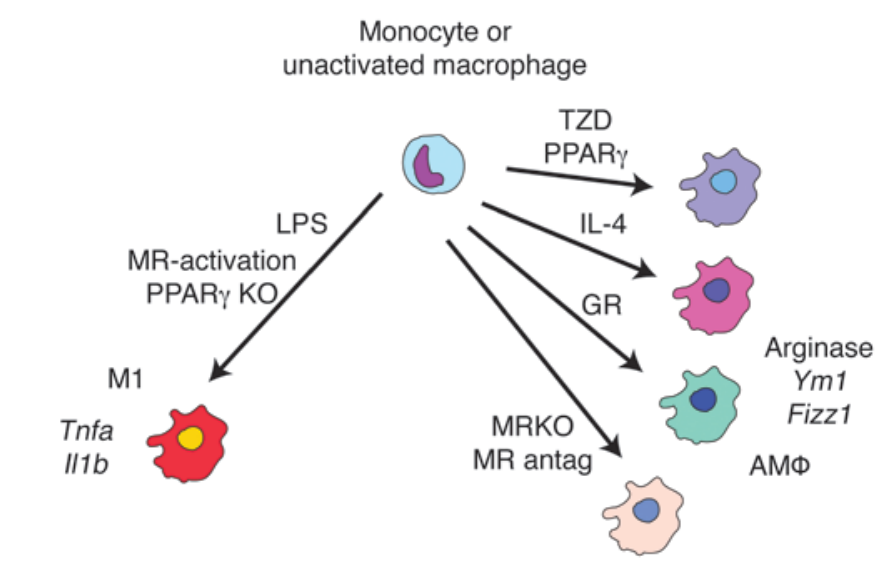

Cardiovascular disease exacerbation
Cardiovascular protection

Figure 11

Nuclear receptor network regulating macrophage polarization. (A) Heatmap of hierarchical cluster analysis of gene expression altered by MyPGKO, IL-4, MyMRKO, and corticosterone treatment in macrophages. Nuclear receptor activation or inactivation can dramatically alter the expression patterns of genes associated with macrophage polarization. Classical activation markers (M1) are segregated from AM $\phi$-associated genes. There are two major subtypes of $\mathrm{AM} \phi$ gene patterns: one where the expression in the different treatments is concordant (AM $\phi 1)$; and one where there are a number of genes where there are substantial differences (AM $\phi 2)$. (B) Summary of macrophage activation and cardiovascular consequences.

circadian rhythm indicates a role for myeloid MR in controlling circadian blood pressure. Since mice are nocturnal, this behavior is similar to nighttime hypertension in humans, referred to as nondipper hypertension. This is an important problem in that it has been linked to risk of congestive heart failure (39). Determining the mechanism may have important implications in humans.

We have identified an MR-dependant transcriptional program that links macrophage polarization with functions associated with ECM structure and TGF- $\beta$ signaling. This is likely an important first step in understanding the molecular signaling that allows macrophages to control hemostatic changes following challenge by high-salt diets or L-NAME. This may also provide a connection between inflammatory changes that occur with obesity and salt-sensitive hypertension. Lumeng and colleagues have shown an important role for macrophage polarization in obesity and adipose inflammation (31).

Despite these increases in blood pressure, there is significant protection from cardiac hypertrophy, demonstrating a direct effect on the cardiac response mediated by the myeloid MR. Most studies using MR antagonists in a variety of different systems have shown decreases in hypertrophy (40-45), although not all of them have (26). This effect goes beyond fibrosis in that it shows effects in suppressing the cardiomyocyte expression of the fetal gene program. Therefore, the effects on hypertrophy are 2-fold, altering the fibrotic and cardiomyocyte response. Cytokines that can be of macrophage origin, such as TNF- $\alpha$ (46) and MCP-1 (47), have previously been shown to cause cardiac hypertrophy. Therefore, these cytokines or others, possibly including inhibitory cytokines, may normally participate in the hypertrophic response, and their alteration provides a possible mechanism for abrogation of hypertrophy in the myeloid MRKO. That non-cardiomyocyte cells contribute to the mechanism of hypertensive cardiac hypertrophy and inflammation has long been appreciated (48). However, this is the first description to our knowledge of a clear role for myeloid cells in cardiomyocyte hypertrophy and the alteration of their phenotype by directed gene inactivation in myeloid cells.

$\mathrm{MR}$ in macrophages opposes other nuclear hormone receptors, PPAR $\gamma$ and GR, which allow endocrine signals to control innate immune responses. Interestingly, this pathway has been targeted by a number of drugs effective in mitigating cardiovascular disease and may represent a common mechanism of action, suggesting that alteration of macrophage polarization is a paradigm for drug discovery. This also raises the possibility that better therapeutic action may be achieved by targeting parallel synergistic pathways in some circumstances. These results identify a previously unsuspected role for MR in innate immunity and open the way for investigations into the function of MR in other peripheral cell types, including adipose tissue and other immune cell types.

\section{Methods}

Animals. Floxed MR mice (22) were initially crossed with LysM-Cre mice (The Jackson Laboratory) to generate MyMRKO ( $M R^{f l f l} ;$ LysM-Cre) mice. Cohorts of FC $\left(M R^{f / f l}\right)$ and MyMRKO mice were generated by crossing $M R^{f l / f l}$ and MyMRKO animals. Genotyping was performed to detect the 
presence of both the floxed and deleted alleles as previously described (22). Heterozygotes were additionally tested as controls and produced no significant results.

MyPGKO and their FC mice were generated by crossing MyPGKO mice (provided by F.J. Gonzalez, NCI, Bethesda, Maryland, USA) with floxed PPAR $\gamma$ mice (49). All animal protocols were approved by the University Committee on Use and Care of Animals of the University of Michigan.

Peritoneal macrophage isolation and treatment. PEMs were isolated 4 days after an i.p. injection of aged 3\% Brewer's thioglycolate as previously describe (50). Macrophages were then cultured in media containing $10 \%$ FBS or 10\% C/D FBS (Hyclone). Resident peritoneal macrophages were isolated from naive animals.

Isolated macrophages were pretreated with either MR agonist or MR antagonist 18 hours prior to stimulation. Classical macrophage activation was stimulated by $100 \mathrm{ng} / \mathrm{ml}$ LPS for 3 hours. Alternative macrophage activation was achieved by $5 \mathrm{ng} / \mathrm{ml}$ of IL- 4 for 24 hours.

Gene expression. Total RNA was isolated using an RNeasy kit (QIAGEN) following on-column DNase digestion. First-strand cDNA synthesis was accomplished using a TaqMan Reverse Transcriptase kit (Roche). QRT-PCR was carried out on an iCycler (Bio-Rad). Relative expression was determined via the Ct method normalized to L32, Gapdh, or $18 s$ standards.

L-NAME/Ang II treatment and blood pressure measurement. Eight- to 10-weekold mice were given a $30-\mathrm{mg} / \mathrm{kg}$ dose of L-NAME accompanied by $0.9 \%$ $\mathrm{NaCl}$ in the drinking water for 14 days. After 10 days of L-NAME treatment, $0.8 \mathrm{mg} / \mathrm{kg} / \mathrm{d}$ Ang II (Sigma-Aldrich) was infused by a subcutaneous osmotic pump (Alzet) for the remaining 4 days. Blood pressures were recorded by telemetry (51).

Cardiac hypertrophy estimation. At the end of L-NAME/Ang II treatment, mice were euthanized and cardiac size was measured as before (52). Ventricular weight to body weight ratio (VW/BW, $\mathrm{mg} / \mathrm{g}$ ) was used as an indicator of cardiac size. Parts of ventricles were either fixed in formalin or snap frozen in liquid nitrogen for further analyses.

Histologic analysis. Parts of ventricles and portions of thoracic aortas adjacent to diaphragm were fixed in formalin, and paraffin sections were stained with $\mathrm{H} \& \mathrm{E}$ and/or $0.1 \%$ picrosirius red. Photomicrographs were analyzed by investigators blinded to the experimental protocol using NIH ImageJ 1.43 (http://rsbweb.nih.gov/ij/) to assess fibrosis and aortic wall thickness (entire circumference). Fibrotic staining was expressed as a percentage of stained areas (red) to the total areas examined.

Immunofluorescence staining. Paraffin sections were first deparaffinized and rehydrated. After boiling in $10 \mathrm{mM}$ sodium citrate buffer to unmask antigens, the slides were blocked in buffer containing normal goat serum, incubated with primary antibody rat anti-mouse Mac2 (eBioscience), and then incubated with fluorochrome-conjugated secondary goat anti-rat antibody (Invitrogen). The results were analyzed using an inverted fluorescence microscope (Olympus).

Affymetrix gene chip analysis. RNA was isolated by RNeasy column and sent to the Affymetrix and Microarray Core Facility of the University of Michigan Comprehensive Cancer Center. RNA quality and concentration were confirmed, and gene chip analysis was done on Mouse 4302.0 Affymetrix chips. Original data were deposited into the Gene Expression Omnibus database (http://www.ncbi.nlm.nih.gov/geo/; accession number GSE23308).

Western blot and plasma corticosterone and aldosterone measurement. Western blot analysis and plasma corticosterone and aldosterone measurement are described in Supplemental Methods.

Statistics and cluster analysis. The results are presented as mean \pm SEM. Pairwise comparisons were done via a 2-tailed Student's $t$ test with correction for multiple genes using Benjamini-Hochberg correction for false discovery rate (53). Multiple comparisons were also tested with a 2-way ANOVA and Bonferroni post-test where indicated. Cluster analysis was performed using uncentered complete linkage clustering using Cluster 3.0 (http://bonsai. hgc.jp/ mdehoon/software/cluster/software.htm). The arrayed expression data were then plotted using TreeView software.

\section{Acknowledgments}

This work was funded by grants R01HL083201 from the National Heart, Lung, and Blood Institute, NIH, and 1-08-RA-137 from the American Diabetes Association (to R.M. Mortensen). S.Z. Duan is a recipient of an American Heart Association postdoctoral fellowship award (0825861G).

Received for publication September 8, 2009, and accepted in revised form June 30, 2010.

Address correspondence to: Richard M. Mortensen, Department of Molecular and Integrative Physiology, University of Michigan Medical School, 7641 Med. Sci. II, 1150 W. Med. Ctr. Dr., Ann Arbor, Michigan 48109-5622, USA. Phone: 734.763.2021; Fax: 734.936.8813; E-mail: rmort@umich.edu. Or to: Sheng Zhong Duan, Key Laboratory of Nutrition and Metabolism, Institute for Nutritional Sciences, Shanghai Institutes for Biological Sciences, Chinese Academy of Sciences, 320 Yueyang Road, Xuhui District, Shanghai 200031, China. Phone: 86.21.54922000; Fax: 86.21.54920291; E-mail: duansz2008@gmail.com.
1. Pitt B, et al. The effect of spironolactone on morbidity and mortality in patients with severe heart failure. Randomized aldactone evaluation study investigators. N Engl J Med. 1999;341(10):709-717.

2. Pitt B, et al. The EPHESUS trial: eplerenone in patients with heart failure due to systolic dysfunction complicating acute myocardial infarction. Eplerenone post-AMI heart failure efficacy and survival study. Cardiovasc Drugs Ther. 2001;15(1):79-87.

3. Guo C, et al. Mineralocorticoid receptor antagonist reduces renal injury in rodent models of types 1 and 2 diabetes mellitus. Endocrinology. 2006;147(11):5363-5373.

4. Lam EY, Funder JW, Nikolic-Paterson DJ, Fuller PJ, Young MJ. Mineralocorticoid receptor blockade but not steroid withdrawal reverses renal fibrosis in deoxycorticosterone/salt rats. Endocrinology. 2006;147(7):3623-3629.

5. Rajagopalan S, Duquaine D, King S, Pitt B, Patel P. Mineralocorticoid receptor antagonism in experimental atherosclerosis. Circulation. 2002;105(18):2212-2216.
6. Guo C, et al. Mineralocorticoid receptor blockade reverses obesity-related changes in expression of adiponectin, peroxisome proliferator-activated receptor-gamma, and proinflammatory adipokines. Circulation. 2008;117(17):2253-2261.

7. Brilla CG, Weber KT. Mineralocorticoid excess, dietary sodium, and myocardial fibrosis. J Lab Clin Med. 1992;120(6):893-901.

8. Funder JW. Mineralocorticoid receptors: distribution and activation. Heart Fail Rev. 2005;10(1):15-22.

9. Martinez FO, Sica A, Mantovani A, Locati M. Macrophage activation and polarization. Front Biosci. 2008;13:453-461.

10. Gordon S. Alternative activation of macrophages. Nat Rev Immunol. 2003;3(1):23-35

11. Stumpo R, Kauer M, Martin S, Kolb H. IL-10 induces gene expression in macrophages: partial overlap with IL-5 but not with IL-4 induced genes. Cytokine. 2003;24(1-2):46-56.

12. Gratchev A, et al. Activation of a TGF-beta-specific multistep gene expression program in mature macrophages requires glucocorticoid-mediated surface expression of TGF-beta receptor II. J Immunol. 2008;180(10):6553-6565.

13. Odegaard JI, et al. Macrophage-specific PPARgamma controls alternative activation and improves insulin resistance. Nature. 2007;447(7148):1116-1120.

14. Odegaard JI, et al. Alternative M2 activation of Kupffer cells by PPARdelta ameliorates obesity-induced insulin resistance. Cell Metab. 2008;7(6):496-507.

15. Kang K, et al. Adipocyte-derived Th2 cytokines and myeloid PPARdelta regulate macrophage polarization and insulin sensitivity. Cell Metab. 2008;7(6):485-495.

16. Ogawa S, et al. Molecular determinants of crosstalk between nuclear receptors and toll-like receptors. Cell. 2005;122(5):707-721.

17. Iglarz $M$, et al. Peroxisome proliferator-activated receptor-alpha and receptor-gamma activators prevent cardiac fibrosis in mineralocorticoid-dependent hypertension. Hypertension. 2003;42(4):737-743.

18. Caglayan E, et al. Differential roles of cardiomyocyte and macrophage peroxisome proliferator-acti- 
vated receptor gamma in cardiac fibrosis. Diabetes. 2008;57(9):2470-2479.

19. Gilmour JS, et al. Local amplification of glucocorticoids by 11 beta-hydroxysteroid dehydrogenase type 1 promotes macrophage phagocytosis of apoptotic leukocytes. J Immunol. 2006;176(12):7605-7611.

20. de Kloet ER, Oitzl MS, Joels M. Functional implications of brain corticosteroid receptor diversity. Cell Mol Neurobiol. 1993;13(4):433-455.

21. Lim HY, Muller N, Herold MJ, van den Brandt J, Reichardt HM. Glucocorticoids exert opposing effects on macrophage function dependent on their concentration. Immunology. 2007;122(1):47-53.

22. Berger $S$, et al. Loss of the limbic mineralocorticoid receptor impairs behavioral plasticity. Proc Natl Acad Sci U S A. 2006;103(1):195-200.

23. Clausen BE, Burkhardt C, Reith W, Renkawitz $\mathrm{R}$, Forster I. Conditional gene targeting in macrophages and granulocytes using LysMcre mice. Transgenic Res. 1999;8(4):265-277.

24. Cho IH, et al. Role of microglial IKKbeta in kainic acid-induced hippocampal neuronal cell death. Brain. 2008;131(pt 11):3019-3033.

25 . Rocha R, et al. Aldosterone: a mediator of myocardial necrosis and renal arteriopathy. Endocrinology. 2000;141(10):3871-3878.

26. Oestreicher EM, et al. Aldosterone and not plasminogen activator inhibitor- 1 is a critical mediator of early angiotensin II/NG-nitro-L-arginine methyl ester-induced myocardial injury. Circulation. 2003;108(20):2517-2523

27. Hevener AL, et al. Macrophage PPAR gamma is required for normal skeletal muscle and hepatic insulin sensitivity and full antidiabetic effects of thiazolidinediones. J Clin Invest. 2007;117(6):1658-1669

28. Bouhlel MA, et al. PPARgamma activation primes human monocytes into alternative M2 macrophages with anti-inflammatory properties. Cell Metab. 2007;6(2):137-143.

29. Bridgham JT, Carroll SM, Thornton JW. Evolution of hormone-receptor complexity by molecular exploitation. Science. 2006;312(5770):97-101.

30. Hu X, Funder JW. The evolution of mineralocorticoid receptors. Mol Endocrinol. 2006;20(7):1471-1478.

31. Lumeng CN, Bodzin JL, Saltiel AR. Obesity induces a phenotypic switch in adipose tissue macrophage polarization. J Clin Invest. 2007;117(1):175-184.

32. Catena $\mathrm{C}$, et al. Insulin sensitivity in patients with primary aldosteronism: a follow-up study. J Clin Endocrinol Metab. 2006;91(9):3457-3463.

33. Rickard AJ, Morgan J, Tesch G, Funder JW, Fuller PJ, Young MJ. Deletion of mineralocorticoid receptors from macrophages protects against deoxycorticosterone/salt-induced cardiac fibrosis and increased blood pressure. Hypertension. 2009;54(3):537-543.

34. Mihailidou AS, Loan Le TY, Mardini M, Funder JW. Glucocorticoids activate cardiac mineralocorticoid receptors during experimental myocardial infarction. Hypertension. 2009;54(6):1306-1312.

35. Avital A, Segal M, Richter-Levin G. Contrasting roles of corticosteroid receptors in hippocampal plasticity. J Neurosci. 2006;26(36):9130-9134.

36. Datson NA, van der Perk J, de Kloet ER, Vreugdenhil E. Identification of corticosteroid-responsive genes in rat hippocampus using serial analysis of gene expression. EurJ Neurosci. 2001;14(4):675-689.

37. De Kloet ER, Derijk R. Signaling pathways in brain involved in predisposition and pathogenesis of stress-related disease: genetic and kinetic factors affecting the MR/GR balance. Ann NY Acad Sci. 2004;1032:14-34.

38. Machnik A, et al. Macrophages regulate salt-dependent volume and blood pressure by a vascular endothelial growth factor-C-dependent buffering mechanism. Nat Med. 2009;15(5):545-552

39. Ingelsson E, Bjorklund-Bodegard K, Lind L, Arnlov J, Sundstrom J. Diurnal blood pressure pattern and risk of congestive heart failure. JAMA 2006;295(24):2859-2866

40. Young MJ, Moussa L, Dilley R, Funder JW. Early inflammatory responses in experimental cardiac hypertrophy and fibrosis: effects of 11 beta\}hydroxysteroid dehydrogenase inactivation. Endocrinology. 2003;144(3):1121-1125.

41. Enomoto S, et al. Effects of eplerenone on transcriptional factors and mRNA expression related to cardiac remodelling after myocardial infarction. Heart. 2005;91(12):1595-1600.

42. Burla AK, Neves MF, Oigman W, Mandarim-de-Lacerda CA. Eplerenone offsets cardiac and aortic adverse remodeling in spontaneously hypertensive rats.
Int J Cardiol. 2007;114(1):64-70.

43. Kobayashi N, et al. Cardioprotective mechanisms of eplerenone on cardiac performance and remodeling in failing rat hearts. Hypertension. 2006; 47(4):671-679

44. Zhang Q, et al. The specific mineralocorticoid receptor blocker eplerenone attenuates left ventricular remodeling in mice lacking the gene encoding guanylyl cyclase-A. Hypertens Res. 2008;31(6):1251-1256.

45. Urabe A, Izumi T, Abe Y, Taniguchi I, Mochizuki S Effects of Eplerenone and Salt Intake on Left Ventricular Remodeling after Myocardial Infarction in Rats. Hypertens Res. 2006;29(8):627-634.

46. Dibbs ZI, et al. Targeted overexpression of transmembrane tumor necrosis factor provokes a concentric cardiac hypertrophic phenotype. Circulation. 2003;108(8):1002-1008.

47. Kolattukudy P, et al. Myocarditis induced by targeted expression of the MCP-1 gene in murine cardiac muscle. Am J Pathol. 1998;152(1):101-111.

48. Graf K, Schaefer-Graf UM. Is smad3 the key to inflammation and fibrosis in hypertensive heart disease? Hypertension. 2010;55(5):1088-1089.

49. Akiyama TE, et al. Conditional disruption of the peroxisome proliferator-activated receptor gamma gene in mice results in lowered expression of ABCA1, ABCG1, and apoE in macrophages and reduced cholesterol efflux. Mol Cell Biol. 2002;22(8):2607-2619.

50. Hoover-Plow JL, Gong Y, Shchurin A, Busuttil SJ, Schneeman TA, Hart E. Strain and model dependent differences in inflammatory cell recruitment in mice. Inflamm Res. 2008;57(10):457-463.

51. Duan SZ, et al. Hypotension, lipodystrophy, and insulin resistance in generalized PPARgamma-deficient mice rescued from embryonic lethality. J Clin Invest. 2007;117(3):812-822.

52. Duan SZ, Ivashchenko CY, Russell MW, Milstone DS, Mortensen RM. Cardiomyocyte-specific knockout and agonist of peroxisome proliferator-activated receptor-gamma both induce cardiac hypertrophy in mice. Circ Res. 2005;97(4):372-379.

53. Benjamini Y, Hochberg Y. Controlling the false discovery rate: a practical and powerful approach to multiple testing. $J R$ Statist $\operatorname{Soc} B$. $1995 ; 57(1): 289-300$ 